\title{
OBSERVATIONS
}

SUR L'AMÉNAGEMENT FRC

\section{DES FORETS,}

ET PARTICULIÉREMENT

\section{DES FORETS NATIONALES.}

Préfentées à l'A Afemblée Nationale, par la Société Royale d'Agriculture, le 9 Juin I79I.

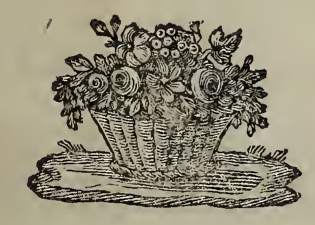

A P A R I S,

De l'Imprimerie de la Feuille du Cultivateur. rue des Foflés Saint-Victor, $\mathrm{n}^{\circ}$. I2. 


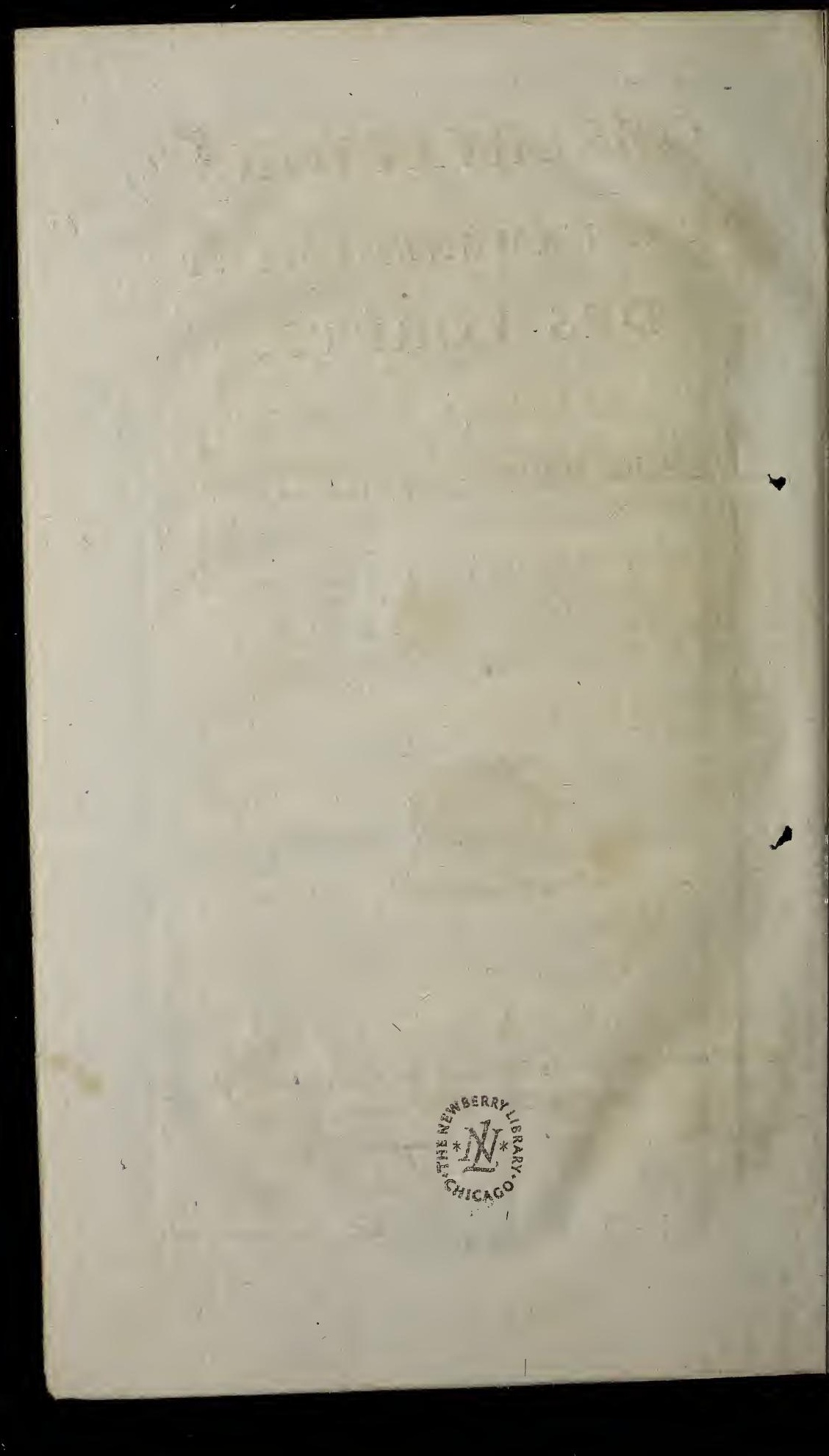




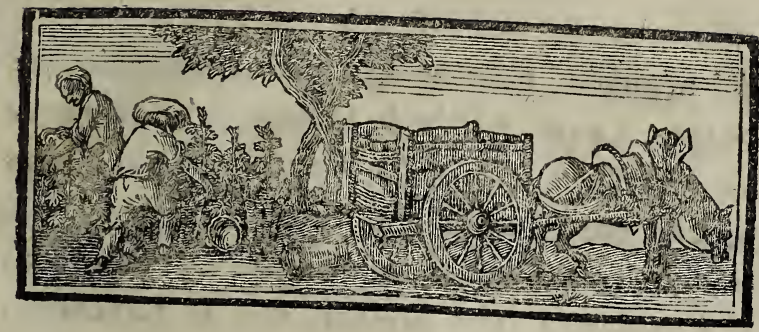

\section{OBSERVATIONS}

SUR L'AMENAGEMENT DES FORETS,

ET PARTICULIEREMENT DES FORETS NATIONALES. Préfentées à l'A Jemblée Nationale, par la Société Royale d'Agriculture.

L'A в ONDANCE du bois eft d'une néceffité fi abfolue, fa confommation fe diverfifie de tant de manières, \& elle eft excitée par tant de befoins fans ceffe renaiffans, qu'il eft incompréhenfible qu'une Société policée ait pu ceffer un feul inftant de veiller à la confervation, à l'aménagement, à la reproduction de fes bois \& de fes forêts. Cependant il n'eft que trop vrai que cette partie importante de la vigilance publique a toujours 


\section{(2)}

écé négligée, pour ne pas dire abandonnée, en France. Le préambule de la fameufe Ordonnance de 1669; fir le fait des Eaux \& Forêts, nous aprend que, jufqu'à cette époque, "le défordre " qui s'étair gliffé dans les Eaux \& Forêts était "fi univerfel \& fi invétéré, que le remède en "paraiffait prefqu impođfible".

La furveillance générale, dont cette loi pofa les principales bafes, était un grand pas vers le but qu'il était effentiel d'atteindre. Nous' devons même regarder comme un commencement d'amélioration, l'avertiffement authentique donné par le Gouvernement, qu'il avait reconnu l'imminence du danger, \& qu'il regardait comme efficaces les mefures quil avait prifes pour en garantir le Royaume. Si dans le choix de ces mefures de détail on ne fut pas auffi heureux que dans celui des bafes principales, ce fut parce qu'il n'était pas du reffort de la pénétration humaine. Le choix, dans ce genre, dépend exclufivement de principes phyfiques qui ne peuvent être faifis \& affermis que par de longues \& favantes obfervations; \& perfonne n'ignore que dans le dernier fiècle on ne foupçonnait même pas à quel point les travaux de celui-ci répandroient de lumière fur les branches de la légillation, dont une faine phyfique doit être l'apui.

Des Phyficiens fe font fucceffivement attachés 


\section{(3)}

à démêler, dans la marche de la Nature, les caufes, nous ne dirons pas de linutilité, mais des inconvéniens inféparables de ces moyens de détail que prefcrit l'Ordonnance de 1669 , pour la confervation \& l'amélioration des forêts. Dès I72I, Réaumur frappé de ces inconvéniens, configna, dans les Mémoires de l'Académie des Sciences, le réfultat de fes obfervations, \& ce réfultat était effrayant: l'inquiétude eft générale, " dit-il, fur le dépériffement des bois du Royaume, "\& peut-être cette inquiétude n'eft-elle que "trop fondée.... Par-tout où il y a des établif" femens de forges, de fourneaux à fer, de "verreries, \&c. on craint que ces établiffe" mens ne tombent par la fuite, faute du bois " néceffaire à leur entretien. Peut-être que l'on " a augmenté la confommation, foit de celui " qui eft employé pour la charpente \& la me" nuiferie, ou façonné pour d’autres ouvrages, " foit de celui qu'on brûle........ L'intérêt de "l'Etat demanderait qu'au moins la quantité du " bois ne diminuât pas pendant que la confom" mation augmente.... Il ne ferait pas raifonna"ble, ajoute-t-il, de fouhaiter que les terres " devenues labourables fuffent remifes en bois; " mais il ferait extrêmement à fouhaiter que les " terrains laiffés en bois", nous donnaffent tout " celui dont nous ayons bejoin pour nos ufages; 


\section{(4)}

* que ces terrains fuffent parfaitement mis en "valeur; \& fur-tout qu'on empếchât leur produit " de diminuer (I). "Réaumur indique enfuite les expédiens qu'on pourrait employer, \& les expériences qu'on pourrait faire pour augmenter les bois du Royaume, \& en quantité, \& en valeur.

Duhamel, Buffon, fe font élancés dans la même carrière. Ils y ont marché en Phyficiens attentifs $\&$ exercés, qui connaiffent tout le poids des obfervations \& des expériences, \& qui favent de combien d'élémens il eft néceffaire de s'affurer pour qu'une expérience foit concluante. M. Télès d'Acofta, M. Pannelier d'Annel, M. Plinguet, fe font occupés du même objet, d'après des effets phyfiques qu'ils ont obfervés. Nous pourrions groffrr cette lifte du nom de plufieurs autres obfervateurs, que leur zèle pourle bien public a couduits dans la même route; mais nous croyons devoir nous borner à citer M. Boncerf, Membre de la Société Royale d'Ágriculture; fon zèle \& fes lumières font connus, \& la Société lui doit un Mémoire dirę fur les fuites fâcheufes qu'ont eu les erreurs en phyfique, dans lefquelles font tombés les Rédacteurs de l'Ordonnance de 1669.

L'importance de la matière ; la notoriété de l'in-

(1) Voyez les Mémoires de l'Académie Royale des Sciences, année 1721 , pag. 284 . 


\section{(5)}

fuffifance des bois qui nous reftent pour répondre à nos befoins; l'impoffibilité d'élever rapidement la reproduction au niveau de la confommation; ont dirigé les premiers regards de la Société fur l'expluitation des mines de charbons de terre, \& fur les tourbières. Ces moyens de fupplément lui ont paru d'autant plus dignes d'attention, que ce font les feuls qui puiffent motiver le retardement des coupes de quantité de bois qui ne font pas encore parvenus à une croiffance fuffifante, \& ce quii eft peut-être plus important encore, les feuls qui permettent d'attendre fans inquiétude le développement complet des plantations, dont la néceffité eft généralement fentie (I)

La Société, conftante dans fa marche fur tous les objets du bien public, liés à la profpérité de l'Agriculture \& de l'économie rurale, a pris des mefures pour fe procurer, par fes nombreux correfpondans au dedans \& au dehors du Royaume, toutes les obfervations qui pouraient la mettre en état de publier un Avis développé fur les femis, les plantations, l'amélioration \& la con* fervation des bois \& des forêts.

(i) La Société Royale d'Agriculture, à qui le Comité d'Agriculture \& de Commerce de l'Affemblée Nationale a fait l'honneur de la confulter, lui a préfenté, au mois de Décembre 1789 , des Objervations fur l'exploitation des Mines de Charbon-de-terre. 


\section{[6]}

Une circonftance d'un ordre majeur, celle de l'aliénation des biens Nationaux, la détermine aujourd'hui à réferver le plan de travail qu'elle fe propofait d'embraffer dans toute fon étendue, \& auquel elle n'a pas renoncé. Elle a regardé comme un devoir de s'attacher dès à préfent aux opérations relatives à la confervation, à l'accroiffement \& à l'aménagement des bois taillis, \& des futaies qui font partie des biens Nationaux.

En conféquence elle nous, a chargés, MM: Lefebvre, Thouin, \& moi, de raffembler les Ouvrages qui ont été publiés fur cette matière, $\&$ les Mémoires manufcrits qu'elle a reçus depuis qu'elle s'occupe du projet de répandre une inftruction générale fur la partie des bois \& des forêts. Après un examen févère, \& de mûres réflexions fur l'ordre à fuivre dans la rédaction de notre travail, il nous a paru que le moyen le plus fimple, le plus expéditif, \& le plus propre à remplir les vies de la Société Royale d'Agriture, ferait de faire imprimer un Mémoire précieux que nous avonstrouvé dans fon dépôt; la première partie de cet écrit traite des taillis, \& l'autre des futaies. Il eft le fruit des lectures, des obfervations, des expériences \& des calculs de M. Varenne, l'un de fes correfpondans. Il nous a paru que l'Auteur. y avait raffemblé tout ce que les écrivains qui lon devancé avaient donné de plus folide fur un 


\section{[7]}

projet $f 1$ intéreffant; qu'il avait fagement raproché les faits qui pouvaient le garantir de quelques méprifes qui leur ont échapé.Ces méprifes, fuffentelles plus nombreufes, feraient pour le moins excufables dans une longue fuite de travaux qui forcent à tout revoir, à tout vérifier, à tout ramener aux principes d'une phyfique fi récemment perfectionnée. Enfin il nous a paru que l'enfemble du travail de $M$. Varenne, indiquait la route la plus profitable à fuivre, foit pour le Public, foit pour les Propriétaires, dans la manutention des futaies \& des taillis. Nous fommes donc perfuadés que la lecture de ce Mémoire juftifiera l'opinion que nous en avons conçue après l'avoir médité.

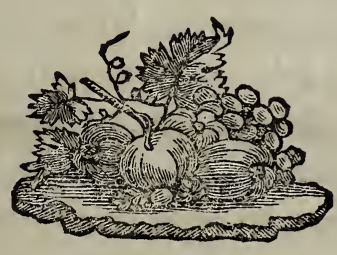

$A_{4}$ 


\section{$M$ É $M$ O I R E}

Sur l'Aménagement des Bois, É particulièrement des Forêts nationales.

PAR M. VARENNE-DE-FENILLE.

PREMIÉRE PARTIE.

Bois taillis.

1E principal objet de la première partie de ce Mémoire, eft de donner une méthode pour déterminer avec précifion l'époque où il eft le plus avantageux de couper les bois, quelle que foit la nature du fond quiles ait produit; \& d'appliquer les réfultats de cette méthode au meilleur des aménagemens dont les bois taillis particuliers \& nationaux font fufceptibles, en fixant à cet égard, toute incertitude.

Cette queftion eft affez importante pour avoir de tout tems occupé les Agronomes, les Légiflateurs, même de grands Phyficiens; \& ce n'eft pas fans une jufte crainte que je hafarde de traiter un fujet fur lequel les Réaumur, les Duhamel \& les Buffon fe font exercés. 


\section{(9)}

Mais après avoir porté, à la lecture de leurs Ouvrages fur les bois, toute l'attention dont j'étais capable, il m'a paru que les principes qu'ils avaient développés, \& les yérités qu'ils avaient établies, ne donnaient néanmoins que des généralités, dont l'application laiffait encore l'Agriculteurpratique dans une fâcheufe incertitude. Aucun de ces célèbres Phyficiens n'a, ce me femble, confidéré la queftion particulière des taillis fous le point de vue que j'ai faif, lorfque je me fuis propofé d'indiquer une méthode démontrée, par laquelle un Propriétaire pourra facilement reconnaître de combien fon taillis, fitué en bon comme en mauvais terrain, aura augmenté chaque année en valeur intrinfèque.

Le vou de Buffon était qu'on la trouvât cette méthode. «En général, a-t-il dit, on peut " s'affurer que dans les bons terrains on ga" gnera à différer la coupe du bois taillis, " \& que dans les terrains où il n'y a pas de fond, " il faut les couper fort jeunes. Mais il ferait $\grave{a}$ " Souhaiter quion pût donner de la précifion à " cette règle, E déterminer au jufte l’âge où l’on " doit couper les taillis. Cet âge eit celui où " l'accroiffement du bois commence à diminuer. "Dans les premières années le bois croît de plus " en plus, c'eft-à-dire, que la production de la " feconde année eft plus confidérable que celle 


\section{(10)}

" de la première année; l'accroiffement de la "troifième année eft plus grand que celui de la " feconde; ainfi l'accroiffement des bois augmente " jufqu'à un certain âge, après quoi il diminue; " c'eft ce point, ce M A X I M U M, quil faut faifir " pour tirer de fon taillis tout l'avantage \& tout "le profit poflible. Mais comment le reconnaître, " comment saflurer de cet inftant? Il n'y a que " des expériences faites en grand, des expériences "longues \& pénibles, des expériences telles que "Réaumar les a indiquées, qui puiffent nous " apprendre l'àge où les bois commencent à " croitre de moins en moins (I)."

Buffon n'était donc pas pleinement fatisfait des expériences indiquées par Réaumur, puifqu'il les trouvait longues \& pénibles. Elles confiftent à faire le choix d'un bois de l'àge de dix ans. On en coupe un arpent qu'on réduit en fagots d'une groffcur \& d'une longueur égale, pour plus d'exactitude on les pèfe. Cinq ans après on coupe un fecond ạrpent à côté du premier : on en pèfe également le produit. Lorfque le premier arpent à repris dix ans d'âge, on le coupe pour la feconde fois, puis pourla troifième fois dix ans après. Enfin on coupe pour la feconde fois le deuxième arpent, celui dont le bois ne doit

(1) Supplément à 1Hiftoire Naturelle, in-40. Ton. 2 。 pag. 257 \& fuir. 


\section{( II )}

être abattu quà quinze ans, \& l'ayant également pefé, on fait la comparaifon exacte du produit d'un taillis coupé trois fois dans trente ans, ou coupé feulement deux fois. Par là, dit Réaumur, on fera en état de juger sil eft plus avantageux de régler les coupes de cel terrain de dix ans en dix ans, ou de quinze en quinze ans.

On ne voit pas quill foit réfervé un troifième arpent à côté des deux autres pour ne le couper qu'à l'âge de trente ans, \& pour en comparer le produit à celui des deux premiers. Peut-être Réaumur s'eft il fait quelque fcrupule de propofer la pefée d'un arpent de bois âgé de trente ans. Quoi qu'il en foit il n'a point diffimulé les ditficultés que fes expériences éprouveraient dans l'exécution. "On ne peut guère efpérer, dit-il, "que l'impatience Françaife permette d'entre" prendre des expériences de fi longue haleine; " nous voulons tout favoir, avoir tout fait dans " le moment. Des expériences de cette nature " feraient auffi plus lùrement conduites par ceux " qui nous gouv'ernent. Elles font un objet " affez important pour l'Etat, pour mériter "leur, attention, \& jofe dire que ce font des "plus belles \& des plus grandes expériences " qu'un Prince puiffe faire entreprendre (I) \%.

(I) Mém. de l'Acad. des Sciences, I72 I. in-12, p. 386 . 


\section{( 12 )}

Ce n'eft pas feulement à raifon de fon extrême difficulté que la méthode de Réaumur me femble peu praticable, \& je crois que l'expérience, comme il la propofe, ferait infuffifante \& fautive.

$I^{\circ}$. Si le plus haut point de croiffance du taillis, ce MAXIMUM que l'on cherche, ne doit fe trouver qu'entre la dixième ou la quinzième année, ou entre la quinzième \& la trentième, comment le reconnaitre?

$2^{\circ}$. En fuppofant que l'expérience ait été faite avec exa\&titude qu'apprendra-t-elle? Le meilleur aménagement du taillis exploité ? C'eft bien peu pour un auffi grand appareil. L'aménagement d'un taillis abfolument femblable ? Mais comment le Propriétaire d'un bois femblable jugera-t-il de leur parfaite conformité?

$3^{\circ}$. Les Maraudeurs, ou quelque accident imprévu, peuvent déranger l'expérience, \& pour peu qu'elle foit altérée, le dommage ne peut plus s'eftimer, la peine eft perdue : il faut recommencer.

$4^{\circ}$. Quel moment choifira-i-on pourle mefurage des fagots \& pour leur pefée, s'il eft vrai, comme on n'en faurait douter, que les bois en fe déffèchant perdent au moins le tiers de leur poids, $\&$ fouvent un quart de leur volume, \& que plus le bois eft en petit volume, plus le deffechement 


\section{( 13 )}

en eft rapide ? La précifion à laquelle Réaumur afpirait eft donc moralement impoffible dans l'expérience qu'il a propofée.

Duhamel eft le Phyficien qui a parlé des taillis avec le plus de détail; mais ce quil en a dit ne conduirait encore qu’à des généralités, quand même fes données, \& conféquemment fes réfultats, ne feraient pas quelquefois contredits.

Cet Auteur eftime (I) que les taillis, effence de chêne, fitués en bon fond, croiffent en hauteur d'un pied chaque année, \& groffiffent de fix lignes annuellement, ou, ce qui revient au même, que chaque couche prend annuellement une ligne d'épaiffeur. Il excepte les baliveaux, qui, fuivant lui, groffiffent de neuf lignes. Il fuppofe enfin que l'arpent d'un taillis de vingt ans contient neuf cents brins.

D'après ces données un arpent, nous dit-il, produira :

\begin{tabular}{|c|c|c|c|}
\hline Années d'âge. & Cordes. & Fagots. & Valeur. \\
\hline 20 ans. & 8 & 800 & $1201 .(2)$ \\
\hline 25 & I 2 & 1200 & I 80 \\
\hline 30 & I 8 & I 800 & 270 \\
\hline
\end{tabular}

(1) Exploitation des Bois, Tom. I, pag. 173 \& fuiv.

(2) Les cercles font entre eux comme les carrés de leur 


\section{( 14 )}

Les obfervations faites à Saint-Dizier, en Champagne, par un grand Maitre des Eaux \& Forêts, M. Tellès d'Acolta (I), ne s'accordent point avec les évaluations de Duhamel. Il ignore, dit-il, dans quelle Province \& dans quel tems les évaluations du tableau de Duhamel ont été faites; mais à Saint-Dizier l'accroiffement des brins de taillis eft de feize lignes de gros, lorfqu'ils font parvenus à 7 à 8 pouces de tour (2)

diamètre. Donc la groffeur du brin de vingt ans eft à la grofteur du brin de 30 ans, comme le carré de 40 lignes, diamètre du brin de 20 ans par la fuppofition, eft au carré de 60 lignes, diamètre du brin de 30 ans. Le carré de 40 eft 1600 , le carré de 60 eft 3600 . Or 1600 lignes font à 3600 lignes comme 120 liv. Sonc à 270 liv. Le calcul de Duhamel eft donc rigoureufement exact; à cela près qu'il a oublié que fon taillis de 30 ans avait cru de dix pieds de hauteur pendant les dix dernières années, \& qu'il a omis de mettre ces dix pieds en ligne de compte. Mais cette extrême exactitude prouve en même tems que fon réfultat n'eft dû qu'au raifonnement \& non à l'expérience.

Tout le refte du chapitre, qui eft fort étendu, ne porte que fur les mêmes bafes, \& ne part que de la même hypothèfe; mais on n'eft pas univerfellement d'accord que cette hypothèfe \& ces bafes foient parfaitement conformes aux loix que fuit la Nature.

(I) Inftructions fur les bois de la Marine. Paris, 1782. in-1 2.

(2) Du moins c'eft ainfi que je l'ai conçu, car la phrafe 


\section{(I5)}

\& fans couper les baliveaux, pour lefquels $M$. d'Acofta témoigne une grande prédilection, contre

n'eft pas parfaitement claire. La voici (pag. 59): « On " a obfervé en Champagne que l'accroiffement des taillis " eft de plus de fix lignes par an, à compter de l'année " où il y a 20 ans faits, \& 7 à 8 pouces de gros. A 30 " ans le taillis a 25 pouces de gros, ce qui fait I pouce 4 * lignes d'accroiffement parannée dans l'efpace de 20 ans \%. Ainfi voilà deux taillis, celui de Duhamel \& celui de M. d'Acofta, tous deux en bon fond, dont l'un ne groffit que de 6 lignes annuellement, lautre de 13.

Ce qui parait plus extraordinaire encore en comparant les deux tableaux, c'eft d'y voir que l'arpent de Duhamel, qu'il fuppofe à 30 ans compofé de brins de 15 pouces de gros, ou cinq pouces de diamètre, donne i 8 cordes \& 18 cent fagots, tandis que l'arpent de SaintDizier, également furchargé de baliveaux, \& portant des brins de 25 pouces de gros, \& 8 pouces un tiers de diamètre, rapporte au plus 24 cordes \& 1200 fagots. Comme les cylindres de même hauteur font entre eux comme les carrés du diamètre de leur bafe, l'arpent fuppofé de Duhamel eft à l'arpent de Saint-Dizier comme le carré de 5 eft au carré de $8 \frac{1}{3}$, ou comme 25 eft à $69 \frac{4}{9}$. Ainfi il eft clair, ou que Duhamel s'eft trompé en partant d'une fauffe fuppofition, ou que l'arpent de Saint-Dizier ne porte pas 900 brins, ou qu'il devait donner au moins 47 cordes.

Les réfultats des deux Auteurs, fur le produit des baliveaux, font, en raifon inverfe, à-peu-près auffi difparates. Ie crois fupperflu de s'y arrêter. 


\section{( 16 )}

l'avis de Réaumur, Duhamel \& Buffon, \& malgré les puiffantes raíons, les obfervations \& les faits dont Buffon fur-tout avait appuyé fon avis. Voici le tableau des produits d'un arpent à Saint-Dizier.

\begin{tabular}{|c|c|c|}
\hline Années d'âge & Cordes. & Fagots. \\
\hline 20 ans. & 14 & 1000 \\
\hline 25 & I7 & I IOO \\
\hline 30 & 20 à 24 & I 200 \\
\hline
\end{tabular}

M. Juge de Saint-Martin, Correfpondant de la Société, donne fur l'accroiffement des taillis un tableau qui diffère \& de celui de Duhamel, \& de celui de M. d'Acofta (I).

Tableau des accroiffemens fuccelfifs d'un arpent pendant quarante ans", extrait de l'Ouyrage de M. Juge de Saint-Martin.

\begin{tabular}{|c|c|c|c|c|}
\hline Années. & $\begin{array}{l}\text { Groffeur } \\
\text { des brins. }\end{array}$ & Cordes. & Fagots. & Valeur. \\
\hline I0 & $7^{\text {pouc. }}$ & 》" & 1000 & $90^{1 .} "$ \\
\hline I5 & $8 \frac{r}{2}$ & " & 1250 & II2 IO \\
\hline 20 & I I & 8 & 500 & $I \neq I \Rightarrow$ \\
\hline 25 & 14 & I2 & 700 & $217 "$ \\
\hline 30 & I5 & I 8 & 950 & 30110 \\
\hline 35 & $\cdots$ & 25 & 1200 & 4I8 \\
\hline 40 & 20 & 26 & $I 200$ & " \\
\hline
\end{tabular}

(I) Traité de la culture du chêne. Paris, 1788. pag. 206 \& fuir. 


\section{( I7)}

Ainfi, fuivant cet Auteur, les couches annuelles feraient moyennement d'une ligne \& demie pendant les dix premières années, de trois cinquièmes de ligne pendant les cinq années fuivantes, d'une ligne depuis I 5 à 20 ans, d'une ligne un cinquième de 20 a 25 ans, d'un cinquième de ligne feulement de 25 à 30 ans, '\& d'une ligne jufte depuis 30 à 40 ans.

Ces faits difcordans, ces différences énormes dans lẹs réfultats, ces qualités mal définies de bon \& de maulvais, données comme abfolues, tandis qu'elles ne font que relatives; ces contradikions répandues malheureufement en trop grand nombre dans nos livres fur l'Agriculture, défolent l'Agronome qui veut étendre fes connaiffances, \& rebutent le Propriétaire peu expérimenté qui cherche à s'inftruire.

Qu'il eût été à défirer, relativement à l'objet qui nous occupe, que Buffon ne fe fùt pas contenté du fouhait qu'il formait pour qu'on donnât de la précifion à une règle fur l'àge où l'on doit couper les taillis! Il n'avait plus qu'un pas à faire pour que fon fouhait fùt rempli par lui même, puifqu'il avaî déjà conçu \& développé cette belle idée du point d'accroiffement, de ce MAXIMUM quil faut faifir pour tirer d'un taillis tout l'avantage poffible. Il ne s'agiffait plus que de chercher un inftrument qui mefurât ces 


\section{( 18 )}

accroiffemens fucceffifs avec exactitude, \& à l'aide duquel on pût déterminer ce Maximum par la voie du calcul.

Mais l'homme de génie confidère l'enfemble de l'objet qu'il médite, fe contente d'en faifir les principaux rapports, les décrit en les généralifant, \& s'arrête rarement fur les détails, à moins qu'il ne fe propofe de s'élever par eux à de grands réfultats. L'Agronome, néceffairement plus timide, parce que la plus légère omiffion peut l'entrainer dans de fortes erreurs, dirige fa marche en fens contraire. Aucun détail ne doit lui être indifférent, \& sil s'élève quelquefois aux grands principes de la Phyfique, ce n'eft que pour y trouver un point d'appui d'où il puiffe defcendre avec fùreté aux moindres particularités de l'Art pratique dont il s'occupe. Mais perfuadé qu'on ne peut s'égarer en fuivant une route que le plus grand Philofophe du fiècle nous à tracée, c'eft après m'être pénétré de fes principes, c'eft d'après fes propres idées, que je vais m'efforcer de réfoudre le problême que lui même a propofé.

Déyeloppement des données du Problême.

Pour plus de clarté nous réduirons la queftion à fes plus fimples élémens. Ainfi je ne confidéterai les taillis que comme bois de chauffage, je 
ferai abitraction de tous les autres ufages auxquels ils pourraient être employés. Je ferai également abltraction des baliveaux, non feulement parce que je les crois plus nuifibles qu'avantageux, \& qu'on a des moyens plus économiques \& plus fürs de fe procurer, en quantité fuffifante, des bois de fervice d'une meilleure qualité; mais parce que cette donnée de plus compliqueroit inutilement la
quefition.

Nous commencerons par examiner ce qui arrive à un tallis fraîchement coupé. Il jette, dès la première année, une quantité innombrable de furgeons, dont à peine il doit fubfifter la centième partie par la fuite. Comme les fouches ont été coupées à fleur de terre, \& qu'il doit fe former de nouveaux yeux, leur développement eft plus tardif que celui des boutons d'un ancien taillis. Néanmoins, \& par la raifon que les racines fourniffent une nourriture furabondante, l'élancement des branches eft plus accéléré, les feuilles font plus larges, leur fanáge eit plus brillant, \& le cours de la fève ne s'arrête qu'à l'approche des premières gelées. Auffi ces jeunes tiges, encore herbacées, font très-fenfibles aux gélées de l'hiver fuivant, $\&$, sil eft rigoureux, il en périt beaucoup.

Lorfque l'entrée du bois eft foigneufement interdite au bétail, lorfque le terrain eft fertile, \& fur-tout profond ; lorfque les fouches font très-rap- 


\section{(20)}

prochées, comme en Breffe, où la plupart des taillis annexés aux domaines fe coupent tous les neuf ans, le bois, dès la quatrième ou la cinquième année eft fi fourré qu'il eft prefque impénétrable.

Cependant, fi vous faites effort pour vous $y$ introduire, vous appercevrez les nuances les plus fortes dans la groffeur des jeunes pouffes : vous en verrez qui varient depuis un jufqu'à quatre \& cinq pouces de tour. A cette époque la partie ligneufe a encore peu de denfité, \& les fortes gélées font toujours à craindre. On doit s'attendre que tous le menu bois fera étouffé par la fuite, foit par défaut d'air, foit plutôt encore par défaut de nourriture.

A l'âge de huit à dix ans le taillis commence de lui-même à s'éclaircir, mais il y refte encore beaucoup de brindilles qui périraient indubitablement, fi l'on en differoit la coupe. J'ai jugé que les principaux brins pouvaient être alors moyennement à la diftance de trois pieds entre eux, \& que l'arpent contiendrait plus de 5000 brins, fi le bétail, le gibier \& les maraudeurs n'y portaient aucun dommage, \& s'il ne s'y rencontroit abfolument aucune clairière; ce qui eft fort rare.

Un taillis de dix ans reffemble à un carré de pépinière dont on n'aurait efpacé les arbres qu'à trois pieds en tout fens. Cet efpace eft fuffifant pour qu'lls y acquièrent fix à fept puces de gros; mais 


\section{(2I)}

paffé ce point ils languiffent \& grofiffent peu, parce qu'ils fe dérobent mutuellement la nourriture. J'en ai la preuve fous les yeux. J'ai dans mes pépinières un carré de frênes, plantés depuis neuf ans. Ceux qui bordent une allée principale, où ils ont pu étendre leurs racines, ont II à I 3 poucés de groffeur, tandis que ceux de l'intérieur n'ont que 6 à 7 pouces au plus.

Il en eft certainement de même d'un taillis. Je m'en fuis convaincu par une épreuve que j'ai faite il y a trois ans. Je venais d'acquérir un petit taillis d'environ deux arpens, effence de chêne, \& attenant ma maifon de campagne. 11 avait fept ans \& il était très-fourré. Je l'ai fait éclaircir : j'ai laiffé fubfifter les plus beaux brins, mais j'ai abattu les brindilles, \& même les brins qui n'étaient pas au moins à la diftance de quatre pieds. J'eftime que ceux que j'ai laiffé fubfifter fon tà fix pieds moyennement, \& que l'arpent en contient 14 à I 5 cents. Je puis affurer que ce taillis a pris au moins cinq pieds d'élévation en trois ans, \& j’ai été fi fatisfait de ce fuccès, que jai fait exécuter depuis de femblables éclaircies fur des taillis plus âgés \& plus étendus ( I ).

(I) Ce fait que je rapporte n’a pas été ma première tentative en ce genre. Dans une terre dont mon père miavait confié l'adminiftration, javais ouvert, en face de la maín, une allée principale, dont l'un des côtés bordait 
Ces éclaircies n'ont tout au plus rendu que le double des frais de l'exploitation, \& cela devait.

une futaie, l'autre un taillis, qu'on était dans l'ufage de couper tous les neuf ans. J'en fufpendis la coupe, \& y fis quelques éclaircies, moins, à la vérité, par des raifons d'économie \& d'un meilleur aménagement, qu'à deffein de donner plus de grace à l'allée. Ce bois peut avoir aujourd'hui quarante ans. Je ne dirai pas qu'il foit, ni auffi élevé, ni auffi beau que l'ancienne futaie, mais je puis dire qu'actueliement les deux côtés de l'allée font. affez d'accord à l'oil pour produire un bel effet.

La futaie dont je parle avait été jardinée autrefois, il s'y trouvait des clairières. Perfuadé qu'elles pouvaient être rétablies par quelque artifice, perfuadé éga'ement que c'eft moins l'ombre qui s'oppofe à la reprife des arbres dont on veut regarnir les clairières, que les racines des arbres voifins, qui, rencontrant une terre fraichement ameublie, s'y jettent avec abondance \& affament le nouvel arbre, $\dddot{j}$ imaginai de ne point planter mes remplacemens dans un creux, mais fur une élévation. A cet effet je faifais tracer au cordeau deux cercles concentriques, l'un de trois, l'autre de quatre pieds de rayon; à l'aide de ce tracél'on creufait autour du centre commun, où l'arbre devait ètre placé, un petit foffé d'un pied de largeur fur douze à quinze pouces de profondeur, \& lon couvrait l'aire du cercle intérieur avec la première couche de gazon extraite du petit foffé. On plaçait l'arbre fur. cette terre remuée, de manière que fes racines fe trouvaient à fix pouces environ au-deffus du fol naturel, qu'on n'avait garde de défoncer. En achevant le folfé on en rejettait les terres fur les racines. L'opération achievée, 
être, vu la jeuneffe du bois \& la difficulté que trouvaient les ouvriers à le façonner \& à le fortir.

l'arbre paraiffait planté fur un cône furbaiffé, ou fur une large taupière. Cette plantation ne me revenait quà environ deux fols \& demi par arbre.

Il faut intercepter le prolongement des racines qui fe rencontrent dans le petit foffé, en les coupant avec foin. Ce que mes Ouvriers exécutaient avec affez d'adrefie, dans ún terrain privé de pierres \& de cailloux, en fervant d'une petite bêche très-tranchante qu'ils nomment un Rochet.

J'effayai de planter de la forte des ormes, des nérifiers, des frênes, des tilleuls, desplatanes, du forbier des oifeleurs, des érables, des châtaigniers, des peupliers \& jufqu“à un tulipier. Les châtaigniers ne reprirent pas, quoiqu'il $\mathrm{y}$ en eut de gros dans la futaie; les peupliers, après avoir pouffé, languirent \& périrent; les frênes, les ormes, les mérifiers \& les platanes réuffirent le mieux. Plufieurs ormes juttèrent des branches de plus de cinq pieds dès la prenière année; mais le tulipier, très-rare alors, y fit des progrès furprenans. J'étais attaché à cet arbre, que j'avais conftamment cultivé moi même,\&que j'avais apporté de Paris dans un pot. Quoique très-petit lorfque je m'avifai de le planter en plein bois, il y arait acquis en peu d'années plus de quatre pouces de diamètre, \& une élévation proportionnée. Quand la terre fut vendue je me réfervai la faculté de l'arracher. J'étais abfent, il le fut mal; il fut tranfporté dans un lieu fec, il y a péri. La plantation que je viens de décrire demande de l'entretien. Si l'on n'a pas foin de rafraichir les foflés ils fe comblent, alors les racines coupées n'en repouffent 


\section{(24)}

Mais les frais \& le produit euffent-ils été au pair, ces ouvriers ont été employés utilement pour euxmêmes, \& ce menu bois, qui auroit péri fur place, \& qui n'eût fervi qu’à retarder l'accroiffement des brins que j'ai laiffé fubfifter, n'a pas été perdu pour la confommation.

Dans quelques années, fi je reconnais que l'épaifeur des couches fe ralentit, je me propofe de faire une feconde éclaircie, \& de donner aux brins la diftance moyenne de fept pieds. Alors l'arpent contiendra réellement les goo brins qua fuppofés

que plus fortement. Les petites buttes exigent aufi d'être labourées tous les ans jufqu'à ce que l'arbre foit fort; non feulement afn qu'il acquière plus d'accroiffement \& de vigueur en moins de tems, mais afin que la terre remuée boive l'eau des pluies. Sans cette précaution l'eau gliffe fur la butte comme fur un toit, fur-tout fi le terrain contient de l'argile, \& l'arbre périt de foif. Dans les lieux bas, au contraire, les eaux féjournent dans le folfé, morfondent les racines, \& la butte fe couvre de mouffe. L'acquéreur peu inftruit de ces précautions in difpenfables, les a néģigées. J'ai revu la fuite de ces épreuves il y a peu d'années; plufieurs buttes fubfiftaicnt encore, quelques arbres plus avantageufement placés que les autres témoignaient de la vigueur, mais la plupart avaient péri.

Quoique cette expérience n'ait qu'un faible rapport avec l'objet de ce Mémoire, j’ai efpéré qu'on voudrait. bien mien paffer le récit dans une note où je l'ai relégué. 


\section{(25)}

Duhamel. Cette coupe fera plus lucrative que la première, parce que le bois fera plus gros, \& que l'exploitation \& la fortie en feront plus faciles.

Enfin, fi, lorfque le taillis aura vingt-cinq ans, on fe contente d'en abattre les trois quarts, il reftera par arpent 225 arbres choifis, bien venans, élancés, vigoureux, qu'on pourra laiffer croître en futaie, en les éclairciffant encore par la fuite, jufqu'à ce qu'ils n'en refte plus que le tiers (I), \& qui remplaceront avec avantage ces baliveaux deftructeurs, auxquels les Officiers des Mâitrifes paraifent li attachés (2).

(I) Une perfonne très-éclairée, fur-tout très-exercée à parcourir les bois, ma afuré qu'elle avait à diverfes fois pris' piaifir à compter le nombre des arbres contenus dans un arpent de belle futaie, \& qu'elle avait trouvé pour terme moyen le nombre 6. Voyez ci-après une méthode plus détaillée d'établir une futaie fur taillis.

(2) On ne fait pas pourquoi ? Si le terrain a peu de fond à quoi bon réferver des balivcaux ? Il n'y croitra jamais un arbre de fervice. Si le terrain a de la profondeur \& que le taillis fe coupe tous les neuf ans, comme en Breffe, quel fervice attendre d'un arbre à tête de pommier fur une tige de ro à I 2 pieds d'élívation? Si les coupes ont été réglées à 20 ans, \& que les tailli ait crû dans un valon ferré quion nomme une combe en Bourgogne, outre qu'une tige de 20 pieds au plus dédommagera taiblement le Propriétaire du terrain qu'elle aura occupé, elle entretiendra fur ce valon une humidité pernicieufe, \& 


\section{(26.)}

Je crois la méthode que je viens de décrire particulièrement applicable aux taillis, qu'on a été jufqu'ici dans l'ufage, en Breffe, de couper tous les neuf ans. A l'égard des bois dont les coupes ont été réglées de tout tems à 20 ou 25 ans, peut-être l'époque de la première éclaircie doit-elle être retardée, peut-être même fera-t-il à propos de n'en faire qu'une, parce que les fouches étant plus féparées, \& les clairières plus fréquentes, il y a plus d'efpace pour nourrir les jeunes brins, \& qu'ily croit par conféquent moins de brindilles.

les effets de la gelée r'en feront que plus funeftes. Les baliveaux ne font donc tolérables tout au plus que dans des taillis que l'on coupe de 30 à 40 ans. Mais dans ce cas, comme ces coupes prolongées annoncent une nature de terre excellente, ce n'eft plus en taillis qu'il eft avantageux de laiffer croitre ce bois, c'eft en futaie. M. d'Acofta, l'un des plus grands partifansdes baliveaux, convient jui même que les Marchands de bois qui approvifionnent Paris, exploitent pour le chauffage, \& tirent peu à la charpente. (Page 69.) Et l'on remarquera que cet Auteur pale d'un canton où le bois groffit de I 6 lignes annuellement. Lorfqu'on fera attention à la très-grande différence entre le prix d'un pied cube de bois de fervice, \& d'un pied cube de bois pour le feu, poura-t-on concevoir comment les adjudicataires des ventes exploitent pour le chauffage \& tirent moins is la charpente, fi les baliveaux font diun auff excellent fervice qte M. d'Acofia le fupjore? 


\section{(27)}

J'en juge par plufieurs tronchées (amas d'arbres étronçonnés) que j'ai fait abattre dans mes domaines pour les transformer en taillis, \& dont je compare les fouches à celles des bois que l'on ne coupe qu'à 30 ou 40 ans. Ceś nouveaux taillis ne font devenus touffus qu'après la feconde coupe, \& je ne me fuis pas bien trouvé d'en avoir éclairci quelques - uns trop-tôt. Car, pour que les brins s'élancent en hauteur, \& groffifient tout à la fois, il faut qu'ils ne foient ni trop, ni trop peu ferrés.

Ces éclaircies doivent fe faire à la journée, non à forfait, en préfence du maître, ou tout au moins d'un homme affidé. Il ne faut jamais abandonner la dépouille qui en provient, aux ouvriers, en déduction du prix de leur travail. Les raifons en font trop fenfibles pour qu'il foit befoin de les expliquer (I).,

(I) Mes Ouvriers employaient une efpèce de ferpe attenant à un manche d'environ fix pieds, qu'il appellent un goyard, \& dont ils fe fervent avec adreffe pour pliffer les buiffons de c'ôture, c'eft-d̀-dire pour les tailler, les rétrécir \& les plier; lorfqu'ils fe voyaient gênés ils eiriployaient la goyarde, autre ferpe plus petite \& qui n'a qu'une poignée. Rarement fe fervaient-ils du hachon \& de la hache. Quelquefois ils faifaient ufage du croiffant, efpèce de faucille armée d'un long manche, lorfqu'il était queftion d'abattre par un coup fec \& afféné, de bas 


\section{(28)}

Mais que les taillis aient été éclaircis ou non, la règle que nous allons établir pour connaitre l'é. poque de leur plus haut point d'accroiffement, leur fera également applicable. On peut feulement prévoir qu'un bois éclairci \& aménagé, comme je viens de le décrire, ne parviendra à fon maximum d'accroiffement, que fortlong-tems après le terme, où un bois abandonné à lui-même, aurait atteint le fien \& commencé à décrô̂tre; parce que chaque bois, fe trouvant plus au large, parcicipera, en quelque forte, à l'avantage des arbres ifolés, dont l'accroiffement, toutes chofes égales d'ailleurs, eft le plus rapide de tous.

Cependant il vient un tems enfin où les brins, qu'elle qu'ait été leur diftance première, font tellement rapprochés les uns des autres, proportionnellement à leur groffeur acquife, que l'efpace qu'ils occupent n'eft plus fuffifant à leur nourriture. Alors néceffairement leur grofiffement commence à fe ralentir, \& diminue enfuite progreffivement; car, fuppofons les brins d'un taillis, de l'âge de 30 ans, à la diftance moyenne de fept pieds entre eux; fuppofons-leur trente pieds d'élévation, \& fept pouces \& demi de diamètre, à raifon de neuf lignes de

en haut, une branche nuifble fús un brin à conferver. Les outils doirent être abondans \& bien affilés fans quoi louvrage languit. 


\section{(29)}

groffiffement moyen par année, on conçoit qu'ils feraient déja trop rapprochés pour ne fe pas nuire réciproquement, \& pour que ceux d'entre eux, qui fe trouveraient d'un tempéramment plus fort, ne finiffent pas à la longue par étouffer les plus foibles; \& c’eft ainfi que s'établiffent la plupart des futaies dans les cantons de réferve. Mais ces futaies feraient incomparablement plus belles \& plutôt formées, fi l'on avait la précaution de les éclaircir, parce que les plus foibles difputent long-tems le terrain, \& ralentiffent, pendant plufieurs années, les progrès des brins vigoureux. Auffi voyons nous, qu'à terrain égal, le groffiffement annuel des arbres d'avenue, ou des arbres crûs en futaies, sils y font fuffifamment efpacés, excède du double \& quelquefois du triple, le groffifement d'un brin crû dans un taillis, où il fe trouve trop gêné, proportionnellement à fon volume.

On reconnaît déjà deux maximum qu'il faut fe bien garder de confondre. Le maximum d'un arbre confidéré individuellement, \& le maximum d'un taillis confidéré en maffe.

Le maximum individuel fe prolonge jufqu'à l'inftant où l'arbre commence à s'altérer dans le cœur. Mais il fera peu queition du maximum individuel dans ce mémoire, puifqu'il ne concerne que les arbres de futaie \& d'avenue. 


\section{( 30 )}

Le maximum d'un taillis, confidéré en maffe, fe fousdivife en fimple \& en "compofé.

Le fimple, qu'on pourrait également nommer maximum phyfique ou abfolu, eft le point où, indépendamment de toute adjonation étrangère, l'accroiffement du taillis commence à décliner phyfiquement.

Dans le maximum compofé il entre une donnée de plus, favoir l'intérêt pécuniaire qu'eût rapporté le prix du taillis vendu, \& dont on eft privélorqu'on differe la vente. Nous ne nous occuperons du maximum compofé, qu'après avoir établi la théorie du maximum fimple.

Théorie du plus haut point d"accroiffement phyfque d'un bois taillis, ou de fon MAXIMUM fimple.

Buffon porte en général le maximum à l'inftant où l'accroiffement du bois commence à diminuer. C'eft le point, dit-il, qu'il faut faifir $r$, pour tirer de fon taillis tout l'avantage poffible.

Je crois que le mot ACCROISSEMENT demande une interprétation, afin d'éviter qu'on ne le confonde avec celui de GROSS ISSEMENT. Car le groffiffement peut avoir commencé de décroître, comme nous l'allons voir, quoique l'accroiffement continue d'augmenter. Rendons ceci fenfible par ur 


\section{(3i)}

exemple, auquel je prie de faire d'autant plus d'attention, que les principes que nous allonsy établir, $\&$ les conféquences qui en dérivent, font la bafe de la réfolution du problême propofé.

Soit un brin de taillis, de l'àge de 20 ans, qui ait groffi de r 2 lignes par année commune.

Sa circonférence fera de 240 lignes, fon diamètre de 80 lignes (environ), \& le carré de ce diamètre de 6400 lignes carrées.

Divifez ces 6400 lignes carrées par 20, le quo. tient vous donnera 320 lignes carrées, nombre qui exprime une quantité proportionnelle à celle dont ce brin a crû moyennement chaque année (I).

Suppofons que le groffiffement de ce brin commence à décroître à cette époque, \& qu'amaigri fucceffivement par le voifinage des brins voifins, il ne prenne plus que I I lignes de gros à la vingt-

(I) Les cercles, \& par conféquent les cylindres de même hauteur, étant entr'eux comme les carrés de leur diamètre, il eft clair que je puis raifonner d'après les carrés des diamètres comme d'après les folides eux mêmes; puifqu ils font proportionnels, les conféquences font abílument les mêmes, les réfultats abfolument femblables; je ne me fervirai même plus par la fuite des mots de quantité proportionnelle, afin d'abréger l'expreffion. Je ne fais cette note que pour les Agriculteurs qui ne feraient pas familiarifés avec les règlẹs de proportion. 


\section{( 32 )}

unième année, to lignes à la vingt-deuxième, 9 la vingt-troifième, 8 lignes à la vingt-quatrième, 7 lignes à la vingt-cinquième, \& $\mathrm{c}$.

Ce brin par la fuppofition aura à 21 ans $25 \mathrm{I}$ lignes de circonférence, où $83 \frac{2}{3}$ lignes de diamètre, donc le carré eft égal à 7000 lignes carrées, (plus une fration que je néglige) mais les cylindres de même hauteur font entr'eux comme les carrés du diamètre de leur bafe; donc le brin de 20 ans eft au brin de 21 ans comme 6400 eft à 7000 .

La différence entre $6400 \& 7000$ eft de 600 . Donc il y a eu à la vingt-unième année beaucoup d'accroiffement, quoique le grofiffement ait diminué; puifque jufques-là l'accroiffement moyen, calculé fur vingt ans, n'avait éré que de 320 \& que nous le trouvons de 600 .

On peut faire un calcul femblable pour chaque année; mais afin d'abréger l'exemple, paffons tout de fuite à la vingt-cinquième année.

Par la fuppofition le brin aura en circonférence, premièrement les 240 lignes qu'il avait à l'âge de 20 ans, plus I I, plus ro, plus 9, plus 8, plus 7 lignes de groffeur acquifes pendant les cinq années fuivantes : total 285 lignes. Son diamètre alors fera de 95 lignes, \& le carré de ce diamètre fera égal à 9025 lignes carrées. Divifez 9025 par 25, vous aurez au quotient $36 \mathrm{I}$, qui. repréfente l'accroiffement moyen pris fur 25 ans. 


\section{(33)}

Sur quoi deux remarques importantes à faire: la première, que malgré la diminution fucceffive dans le groffiffement, l'accroiffement moyen eft néanmoins plus fort quill ne l'était à l'âge de 20 ans, puifquil n'était alors que de 320 lignes, $\&$ que nous venons de le trouver de $36 \mathrm{r}$.

$2^{\circ}$. Que l'accroiffement total était repréfenté à l'âge de 20 ans par le nombre 6400 , \& cinq ans après par le nombre 9025 ; différence trèsforte, \& qui montre déjà l’avantage qui fe trouve à avoir différé la coupe.

A la vingt-fixième année, le brin, par la fuppo: fition, aura 29r lignes de circonférence, 97 lignes de diamètre; donc le carré eft égal à 9409 lignes carrées.

La différence entre 9025 carrẻ du diamètre d'un brin, fuppofé à 25 ans, \& 9409 , carré de ce même brin fuppớé à 26 ans, n'eft plus que de 374 lignes carrrées. Mais l'accroiffement moyen de ce brin, à l'âge de 25 ans, était de 36 I lignes; done il n'y a plus de bénéfice à fufpendre la coupe; donc à l'âge de 25 ans il avait acquis, à fort peu de chofe près, fon plus haut point d'accroiffement (I).

(I) On conçoit que fil le déclin du groffffement fe fait avec plus de lenteur, le maximuin fera neceffairement prolongé. Par exenple, en fuppofant que le groffffement fe foit 


\section{( 34 )}

Cette démonftration eft la bafe de ce qui me refte à dire. Ce qui a été démontré à l'égard d'un feul brin, eft applicable à tous les brins à la fois qui compofent un arpent de taillis, quel que foit le groffiffement que l'on fuppofe à chacun d'eux, \& quel que foit l'âge du bois. Les données \& conféquemment les réfultats peuvent être différens, mais les principes, mais la formule du cacul font \& demeurent effentiellement les mêmes.

Tous les brins d'un taillis ne fe reffemblent pas fans doute, ils font inégaux en groffeur; auffi ne propofé-je pas de juger de tout un taillis par un feul individu. Mais on en jugera par une approximation

maintenu uniformément jufquà lầge de 20 ans, \& qu’à dater de cette époque il n'ait décliné que d'une demiligne par an, on demande à quel ấge ce taillis aura acquis fon maximum? Le calcul démontrera que c'eft à 33 ans. Car, Par l'hypothèfe la circonférence a 32 ans d'âge $=240$ $+105=345$.

Le diamètre = II 5 .

Le carré de $115=13225$.

13225 divifé par $32=413$. Ce dernier nombre repréfente l'accroiffement moyen pendant 32 ans.

A 33 ans d'âge la citconférence $=35 \mathrm{c} \frac{\mathrm{T}}{2}$.

Le diamètre $=$ I $6 \frac{5}{6}$.

Le carré du diamètre $=13650 \frac{1}{36}$.

La différence de 13650 à 13225 eft de 425 , qui séloigne très-pèu de 413 , accroifiement moyen des 32 années précédentes. 


\section{(35)}

qui s'éloignera très-peu de l'exactitude rigoureufe, fi l'on choifit un certain nombre de brins dans les différentes claffes de groffeur, \& les différentes efpèces d'arbres qui forment l'effence du taillis, pour en former une moyenne proportionnelle, \& la foumettre au calcul d'après la formule fuivante.

\section{I.}

Choifffez 20 brins, ou en tel nombre que vous voudrez. Vous les défignerez, numéroterez $\&$ décrirez de manière qu'on puiffe aifément les reconnaître aux années fuivantes.

\section{I.}

Mefurez le diamètre de chacun d'eux, au moyen du compas courbe ( $p l$. ci-jointe). Prenez votre mefure conftamment à la même hauteur, à trois pieds, par exemple; \& parce que les arbres ne font jamais parfaitement ronds, mefurez-les par leur plus grand diamètre : l'opération s'en fait plus facilement.

\section{I I.}

Carrez chacun de ces diamètres.

$$
\text { I V. }
$$

Additionnez les 20 produits : formez-en un total.

$$
\text { V. }
$$

Divifez ce total par le nombre de brins choifis.

$$
\mathrm{V} \text { I. }
$$

Divifez le quotiènt de votre première divifion $\mathrm{C}_{2}$ 


\section{( $\left.3^{6}\right)$}

par le nombre des années du taillis. Ce dernier nombre, ou fecond quotient, vous donnera la moyenne proportionnelle, ou croiffance moyenne du taillis, pendant les années qui ont précédé le mefurage.

\section{I I.}

Recommencez la même opération une ánnée après, \& à la même époque. Comparez les deux quotients de l'article 6 . Leur différence vous donnera jufte l'accroiffement du taillis pendant la dernière année.

$$
\text { EXE M P L E. }
$$

Soit un taillis, âgé de 15 ans, dontil s'agit de connaître l'accroiffement pendant la feizième année.

(I). On choifira cinq brins parmi les petits, dix parmi les moyens, cinq parmi les grands.

(2). (3). (4). Suppofons que le mefurage donne, favoir.

\begin{tabular}{|c|c|c|}
\hline Numéros. & Diamètre. & $\begin{array}{c}\text { Carré du diamètre } \\
\text { réduit en lignes } \\
\text { carrées. }\end{array}$ \\
\hline I & 30 lig. & 900 \\
2 & 29 & 841 \\
3 & 28 & 784 \\
4 & 31 & 961 \\
5 & 32 & 1024 \\
6 & 35 & 1225 \\
7 & 33 & 1089 \\
8 & $34 \frac{5}{2}$ & $1190 \frac{\pi}{4}$ \\
\hline
\end{tabular}




\section{(37)}

\begin{tabular}{|c|c|c|}
\hline Numéros. & Diamètre. & $\begin{array}{c}\text { Carré du diamètre } \\
\text { réduit en lignes } \\
\text { carrées. }\end{array}$ \\
\hline & $\begin{array}{c}\text { Ci-contre. } \\
\text { 9 }\end{array}$ & $8014 \frac{1}{4}$ \\
I0 & 36 & I296 \\
I1 & 34 & II 56 \\
I2 & 37 & I369 \\
I3 & $36 \frac{1}{2}$ & $1332 \frac{1}{4}$ \\
I4 & 38 & 1444 \\
I5 & $39 \frac{1}{2}$ & $1482 \frac{1}{4}$ \\
I6 & 36 & 1296 \\
I7 & 44 & 1936 \\
I8 & 45 & 2025 \\
I9 & $46 \frac{1}{2}$ & $2116 \frac{1}{4}$ \\
20 & 47 & 2209 \\
& 48 & 2304 \\
\hline
\end{tabular}

(5). Divifez 27980, par 20 nombre des brins choifis, le quotient I 399 , peut être confidéré comme moyenne proportionnelle, ou le brin moyen, de tous les brins du taillis.

(6). Enfin, divifez I399, par I5 nombre des années du taillis, vous aurez, pour quotient $93 \frac{4}{15}$, \& ce dernier nombre exprimera le groffiffement moyen du taillis, pendant chacune des 15 années.

(7). Recommencez un femblable mefurage, fur les mêmes numéros, l'année fuivante, \& fuppofons que chaque numéro ait groffi, pendant la feizième année, conformément à la table fuivante.

$$
\text { C } 3
$$


$\left(3^{8}\right)$

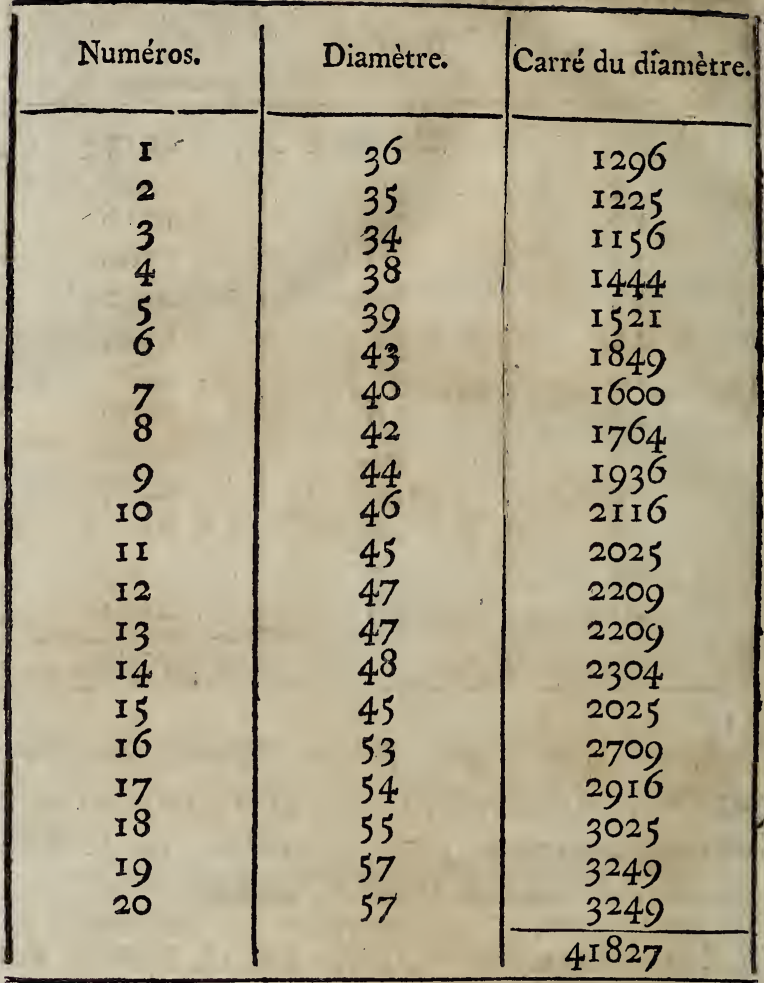

41827 divifés par 20 nombre des brins, donnent au quotient $209 \mathrm{I} \frac{7}{20}$; qui, divifés par 16 nom. bre des années, donnent au quotient $130 \frac{1}{16}$.

Donc l'accroiffement de la feizième année excède l'accroiffement moyen des quinze premières années dans le rapport, (en négligeant les frae- 


\section{( 39 )}

tions), de I30 à 93; c'eft-à-dire, de plus d'un tiers en fus.

Continuez chaque année les mefurages, jufqu'à ce que le cacul prouve qu'il n'y a prefque plus de différence entre le dernier accroiffement \& l'accroiffement moyen, pris fur toutes les années précédentes; alors, le taillis fera parvenu à ce point, à cet inftant, paffé lequel il n'y aurait prefque plus que de la perte à en différer la coupe.

\section{Théorie du Maximum compofé.}

Nous ne nous fommes occupés jufqu'ici, que du maximum fimple, abfolu, phyfique, tel que la nature le donne. Mais nous avons annoncé, page 30 , qu'il y avait un maximum compofé, \& que dans celui-ci, il entrait une donnée de plus, à laquelle il falloit avoir égard.

Un Propriétaire ne coupe pas toujours fon bois pour le confommer : plus ordinairement il ne l'abat que pour le vendre. S'il diffère, il perd l'intérêt du prix de la vente; \& s'il diffère pendant plufieurs années, cesintérêts s'accumulent \& deviennent confidérables. Il eft donc queftion de s'affurer, par le moyen du cacul, fi la mieux value qu'il obtiendra, en différant fa coupe, le déciommagera furabondamment de la perte qu'il eft dans

$\mathrm{C}_{4}$ 


\section{( 40$)$}

le cas de faire fur les intérêts, \& comment il doit agir en économe attentif.

Rendons l'explication de cette hypothèfe fenfible par un exemple, \& pour réfoudre ce nouveau problême, nous prendrons, pour la moyenne proportionnelle d'un bois taillis de $I 6$ arpens, un brin qui groffiffe uniformément de 4 lignes de diamètre par an. Nous fuppoferons la valeur de ce taillis égale à $\mathrm{I} 600$ liv. à raifon de Ioo liv. pararpent, de l'âge de ro ans.

La première colonne (table première), marque le nombre des années du taillis.

La feconde colonne, donne l'accroiffement fucceffif du diamètre de la moyenne proportionnelle, à raifon de 4 lignes par an.

Le carré de chacun de ces diamètres eft calculé dans la troifième colonne, \& chaque ligne carrée, par l'hypothèfe, équivaut à 20 fols.

La quatrième colonne indique la quantité de lignes carrées, dont le carré du diamètre a augmenté chaque année, fur l'année précédente.

La fixième colonne exige une explication. Puifque le taillis valait, par l'hypothèfe, I600 là la fin de la dixième année, il eft clair que fa production a été, par année commune, de 1601 .; \& que sil eût été coupé \& vendu à dix ans, le terrain eût acquis, à la onzième année, une valeur égale à ces mêmes I6oliv. Or, on fe prive de cette va- 


\section{(4I)}

leur en s'abftenant de le couper à la fin de la dixième année. Voilà ce que j’ai appelé ( colonne 6); valeur perdue, par la non-reproduction.

Cette perte s'exprime par le quotient d'une divifion, dont la valeur acquife par le bois, depuis $\mathrm{fa}$ dernière coupe, eft le dividende, \& le nombre des années qu'il a vécu, le divifeur. Ainfi, dans l'hypothèfe, à la douzième année, cette perte eft exprimée par 176, quotient de 1936 , (valeur du taillis à l'âge de I I ans ) divifés par I I. Semblablement à la treizième année, cette perte eft exprimée par 192, quotient de 2304, divifé par 12, ainfi des autres.

Mais cette perte, ou plutôt cette déduction, commune au maximum fimple \& au maximum compofé, eft encore augmentée dans le maximum compofé, par la perte des intérêts du prix de la chofe non-vendue. Ce font ces intérêts dont il eft fait mention dans la colonne 7 ; ils augmentent, comme on voit, en proportion de ce que la valeur du taillis a augmenté pendant toutes les années qui ont précédé l'année quelconque d'où l'on part pour fupputer cette valeur.

La colonne 8 , donne le total des deux pertes prifes enfemble.

Enfin la colonne 9, fait la balance de la mieux value de l'accroiffement annuel (mentionné à la colonne 4 ), avec le total de la ferte. 


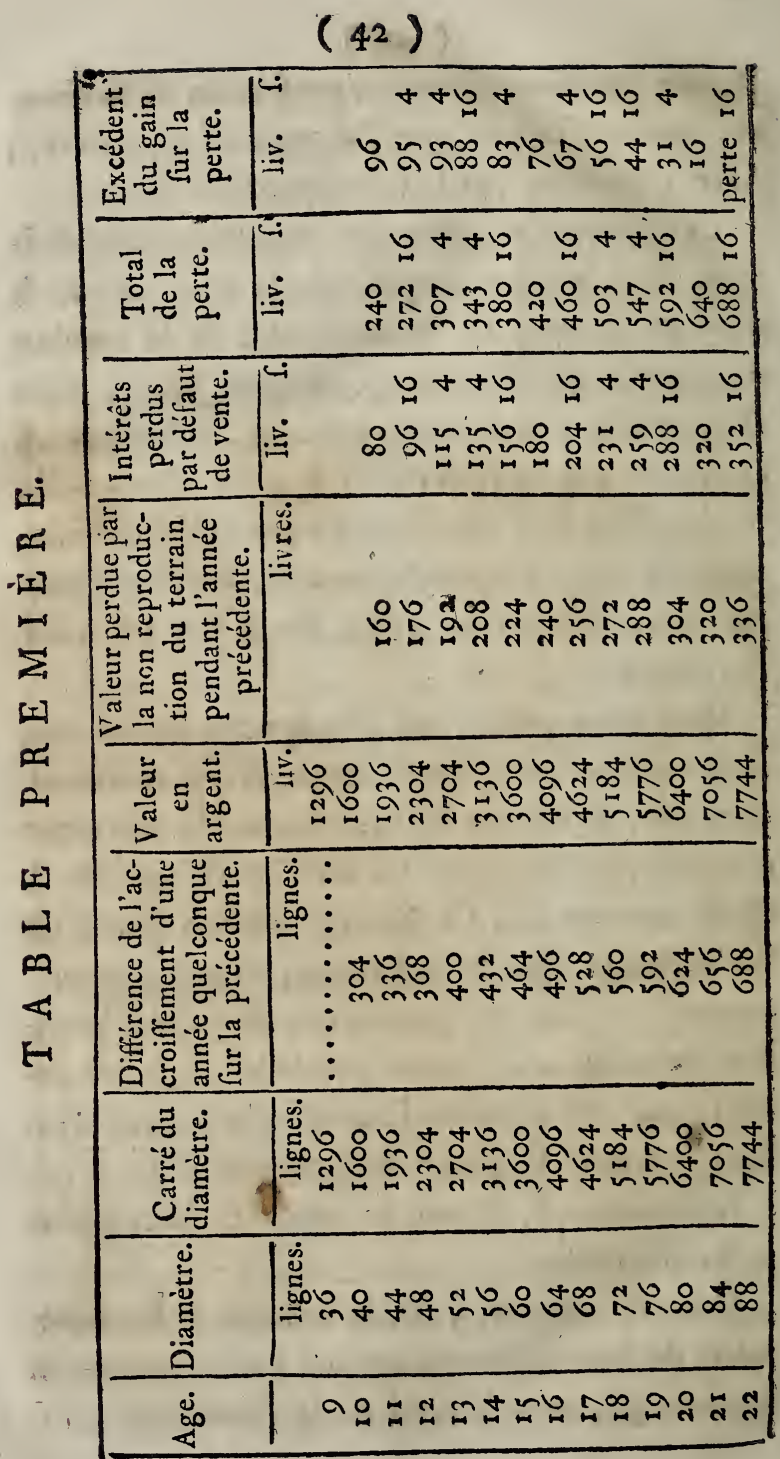




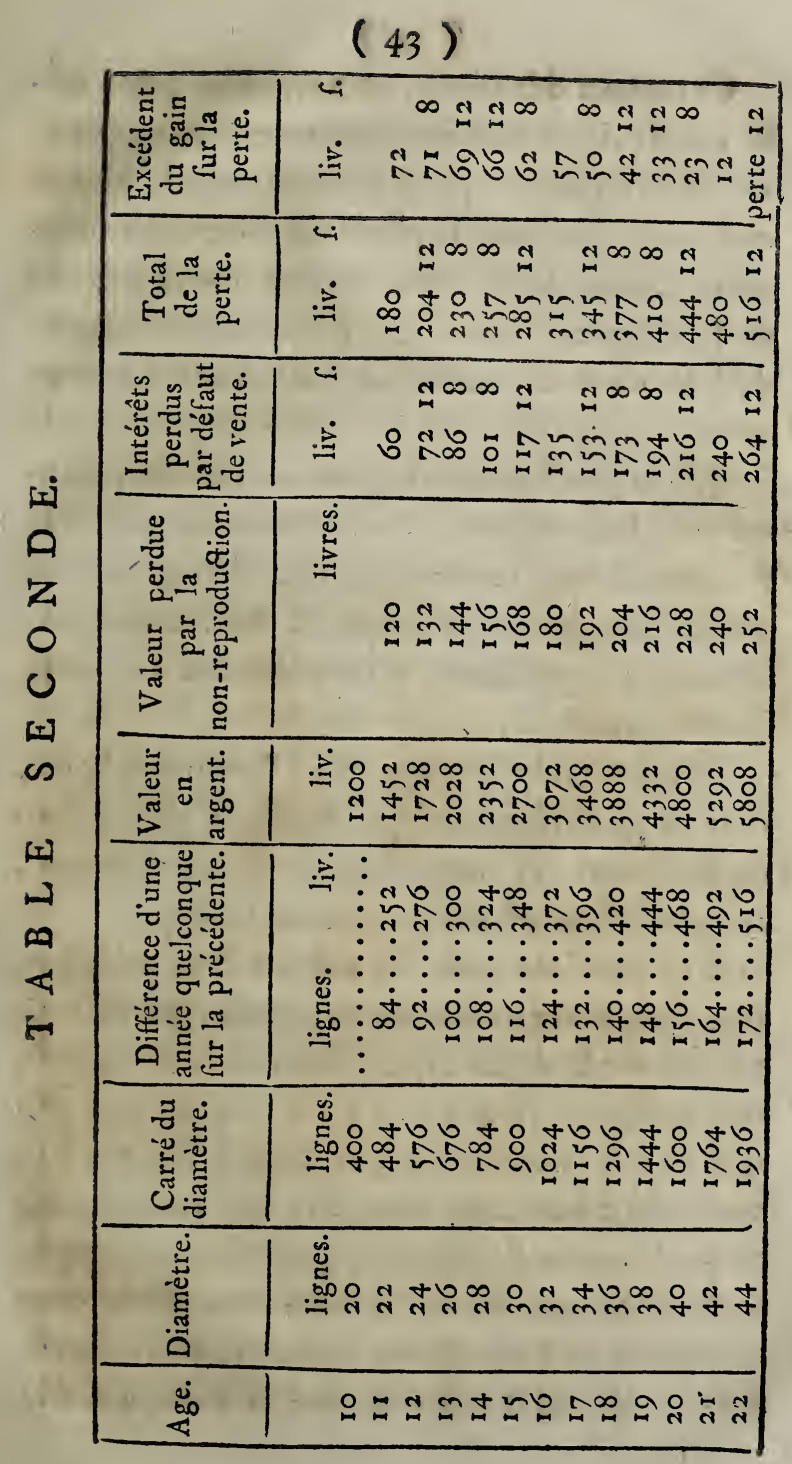




\section{(44)}

Ce tableau démontre, qu'à fuppofer qu'un taillis croiffe d'une manière uniforme, fans augmenter, ni diminuer fon groffiffement annuel, alors le maximum compofé, le plus haut point d'accroiffement utile que l'on cherche, fe trouve à la fin de la vingt-unième année, puifqu'en ne coupant qu'à la vingt-deuxième on commence à être en perte.

Que le groffiffement foit lent ou prompt, pourvu qu'il foit uniforme, le maximum compofé n'en portera pas moins conftammentfur la vingt-unième année. Les mêmes principes \& les mêmes conféquences s'appliquent à l'une comme à comme l'autre hypothèfe.

Voyez la table feconde, où , à l'uniformité près, dans le groffiffement de la moyenne proportionnelle, toutes les autres données font différentes, mais le réfultat eft abfolument femblable.

D'où provient donc cet axiome \& ce principe, dont l'expérience confirme la jufteffe, que les coupes doivent être plus rapprochées dans les mauvais terrains que dans les bons? C'eft uniquement parce que dans les mauvais fols, les brins s'affament nuutuellement beaucoup plus vite que dans les fols riches \& profonds, \& que le groffiffement des tiges ceffe beaucoup plutôt d'être uniforme. Dans cette occurence, un Propriétaire attentif pourra fe fervir, avec fuccès, de la méthode que j’ai indiquée, afir 


\section{(45)}

d'étudier \& de reconnaître, par des mefurages, le temis où il lui convient de couper.

Mais il n'en eft pas moins vrai \& démontré, que, tout excellent que foit un terrain, le maximum utile au Propriétaire qui veut vendre, ne fe prolonge pas au-delà de la vingt-unième année, à moins, qu'au moyen des éclaircies dont j'ai parlé, le grofffffement, après 20 ans, loin de fe ralentir, augmentât; qu'au lieu, par exemple, de continuer d'être de 12 lignes moyennement, il s'élevât à I 4 ou à I6. Cela peut arriver; il y a même des probabilités que cela arrivera; mais l'expérience peut feule nous éclairer à cet égard, \& je ne crois pas qu'elle ait été faite (I).

(1) Voici cependant une obfervation qui annonce qu'à cet égard mon opinion n'eft pas hafardée.

J'ai fait abattre en Novembre 1789 , un orme de l'âge de 39 ans, pour des expériences relatives à mon travail fur les qualités individuelles des bois. Cet orme avait cru dans un très-bon fond, autrefois en futaie. Les arbres de cette futaie étant clair-femés, d'une groffeur très-inégale, \& plufieurs d'entr'eux étant fur le retour, je fis, en $177^{6}$, une coupe blanche \& ne réfervai pour baliveaux que quelques jeunes ormes. Ce canton, d'environ 25 arpens, eff devenue un excellent taillis: L'orme dont je parle avait été gêné vraifemblablement dans fa première croiffance; le lendemain du jour où il fut abattu, jen fis couper une tranche perpendiculairement à fon 2xe, pour connoitre fon âge, \& obferver de combien 


\section{$(46)$}

L'ufage prefque univerfellement fuivi dans le Royaume, par les grands Propriétaires, de régler la coupe de leurs taillis à 20 ans, séloigne donc fort peu, comme on voit, de ce qu'annonce notre théorie, par rapport au maximum compofé. Il me femble que cela devait être. Des calculs par approximation, fouvent répétés, ont dû naturellement conduire à des réfultats peu différens de ceux que nous avons rigoureufement démontrés.

Remarques particulières \& réponfes à quelques

\section{objections.}

Les années ne font pas également favorables à

ferait fa retraite. La tranche portait is pouces à fon plus grand, \& 12 pouces 7 lignes à fon plus petit diamètre. La retraite caufée par le deffèchement qui n'eft pas encore complet, eft de 3 lignes fur chacun de ces diamètres. Mais il eff fingulièrement remarquable que le grand diamètre avait augmenté en $\mathrm{I} 3$ ans de 8 pouces 4 lignes, $\&$ le petit de 5 pouces II lignes, tandis que la grofieur acquife pendant les 26 années précédentes n'a été que de 6 pouces 5 lignes, tant à l'égard du grand que du petit diamètre. Ainfi le groffiffement moyen des 26 premières années n'a été que d'un peu moins de 9 lignes par an, \& celui des I 3 années fuivantes a été de près de 20 lignes. On ne peut douter que ce fubit accroiffement ne foit dû à la liberté de s'étendre qu'on eu les racines de l'arbre, depuis que la futaie a été abattue. Et ce que dit Duhramel fur l'accroiffement áccéléré des baliveaux, confirme cette opinion ; ( voyeźz page Iz de ce Mémoire ). 


\section{(47)}

Ia végétation, \& leur viciffitude influe néceffairement fur l'épaiffeur des couches ligneufes. Divers accidens, un été froid \& pluvieux, ou fec \& brûlant, un déluge d'infectes, \&c. peuvent déranger la marche ordinaire de la nature; mais les gelées du printems font l'événement le plus à redouter pour les taillis. J'ai obfervé, en I 790 , à quel point ces gélées étaient capables de nuire à l'accroiffement des arbres.

Un quinconce de tilleuls, que je vois tous les jours, eft planté, depuis 20 ans, fur une terraffe, àl'expofition du levant \& du midi. Parmi ces tilleuls il y en a de précoces, d'autres, \& en particulier deux d'entre-eux, font très-tardifs. Sur la fin d'Avril, il eft furvenu une gélée de trois degrés; les feuilles fe font rembrunies, leur organifation a été dérangée, \& leur développement, ainfi que celui des jeunes pouffes, a été tellement arrêté, qu'aucunes des ces feuilles, mêmes celles qui ne fe font épanouies que depuis la gélée, n'ont acquis leur volume ordinaire. Elles font tombées en partie aux mois de Mai \& Juin; une partie a fubfifté pendant l'automne. Les branches de l'année font demeurées chétives. Les tilleuis tardifs, au contraire, n'ont point fouffert, \& ont été très-touffus. Heureufement, le chêne eft moins hâtif que le tilleul.

En mefurant les arbres, pour en étudier le maximum, il convient, par conféquent, d'avoir égard 


\section{$\left(4^{8}\right)$}

à ces événemens particuliers, fans quoi, d'après les mefurage, on pourrait prendre pour une décroiffance permanente, une décroiffance qui ne ferait qu'accidentelle.

Les expériences que j’ai faites, fur les qualités individuelles des bois, m'ont mis dans le cas d'examiner les branches de plufieurs arbres coupées perpendiculairement à leur axe : j’ai fouvent obfervé une couche annuelle, fort étroite, entre deux couches épaiffes. Cette différence provient fans doute de quelques-uns des accidens dont je viens de parler. Quelquefois encore, j’ai vu des féries de ro à 12 couches, affez minces, précédées \& fuivies de couches plus larges. On ne peut guère, ce me femble, attribuer la maigreur des unes, \& l'embonpoint des autres, quà un voifin incommode, dont l'arbre fe fera enfin débarraffé.

L'expérience, telle que je l'ai propofée, peutêtre déconcertée par un accident imprévu : un des brins deftinés au mefurage peut avoir été écorcé pour en faire de la teinture, ou coupé par un malfaiteur; mais le remède, ce me femble, eft à côté du mal : on lui fuppléera un autre brin, d'un calibre femblable, ou bien on le rayera de tous les calculs précédens; \& le feul inconvénient qui puiffe en réfulter, fera d'avoir une moyenne proportionnelle, calculée fur I9 (dans l'hypothèfe ), au lieu de l'être fur 20. 
Si je n'ai eu aucun égard dans mes calculs, à la hauteur que le taillis acquiert par fucceffion d'années, ce n'eft pas par oubli; mais j'ai cru pouvoir faire entrer cet accroiffement en compenfation avec la quantité, affez confidérable, de petits brins étouffés fous la maffe des tiges plus vigoureufes. Cependant, tout a un terme : il fubfifte affez peu de brindilles dans un taillis de vingt ans; en y voit encore, à la vérité, quelques brins faibles, mais qui réfiftent pendant plufieurs années : à cette époque ce ne font guère que les branches inférieures d'un jet vigoureux, qui fe déffèchent fur la tige. Ainfi, paffél'âge de 20 ans, l'accroiffement d'un taillis, en hauteur, pourrait entrer comme donnée utile dans le calcul; mais cette donnée ne ferait qu'ajouter encore au bénéfice de la prolongation des coupes.

Quoique jaie dit, au commencement de ce Mémoire, qu'en traitant des bois taillis je ne les confidérerais que comme bois de chauffage, il n'en eft pas moins certain que, dans un taillis de 32 ans, il fe rencontrera des, tiges qui porteront fix à fept pouces d'équarriffage.

Plus un taillis eftâgé, plus la quantité relative de l'aubier diminue, \& celle du bois parfait augmente. Il n'y a prefque que de l'aubier dans un taillis de dix ans.

Plus les couches annuelles font épaiffes, \& plus, 


\section{( 50 )}

toutes chofes égales d'ailleurs, le bois acquiert de force, de denfité, de dureté, \& moins les couches d'aubier font nombreufes (I). Il eft donc avantageux de faire des éclaircies, puifqu'elles favorifent le grolfiffement; \&, ne ferait-ce pas la même caufe, linfuffifance d'alimens, qui, après avoir produit le retréciffement des couches, produirait auffi cette faibleffe des fibres ligneufes?

Plus un taillis eft jeune, plus il eft expofé aux funeftes effets de la gelée, de la grêle, aux dégats du bétail, de la bête fauve, \&cc. On pare à une partie de ces accidens en prolongeant les coupes; la dépenfe, pour le rétabliffement de la clôture, revient auffi moins fréquemment.

Lorfque d'auffi puiffans motifs s'oppofent à l'anticipation des coupes, comment le pernicieux \& intolérable ufage d'abattre les bois à l'àge de neuf ans, s'eft-il introduit \& perpétué en Breffe? En voici, je crois, les raifons.

Autrefois, cette Province était moins peuplée qu'elle ne l'eft aujourd'hui; la confommation en bois de chauffage était donc moindre.

Les futaies partielles y étaient cercainement plus abondantes, puifque l'on voit encore que la plupartdes habitations anciennes, tant urbaines que ruftiques, font conftruites en bois de charpente.

(I) Voyez les Mémoires de Buffon \& Duhamel。 


\section{( 5 I )}

La Breffe a été longtems fans commerce, \& prefque inabordable en hiver : le bois de fervice ne s'exportait pas, faute de débouchés; mais depuis la confecion des routes, il a été façonné en bois carrés \& en merrain, \& s'eft exporté.

Enfin, les défrichemens, encouragés par le haut prix des grains, ont diminué, confidérablement la quantité des terrains précédemment occupés par les bois; de forte que la confommation a augmenté en raifon inverfe de la reproduction.

Il y a peu de grandes forêts dans cette Province On n'y compte guère que celles qui lui ont été confervées par les maifons religieufes; car les bois du Roi ne montent pas à plus de 684 arpens, \& font très-déterriorés.

Quelques Privilégiés avaient confervé des bois dans leurs terres, plufieurs Particuliers en poffèdent encore; cependant on ne peut, raifonnablement, donner à ces pofieflions le nom de forêts; $\&$, dans ces derniers tems, ces poffeffions ont été en partie dévaftées.

De tout tems il y a eu une fort grande quantité de boquetaux, communément de cinq à fix, rarement de quinze à vingt arpens, annexés à prefque tous les domaines, faifant partie de la ferme, \& deitinés au chauffage \& aux autres ufages du Fermier. Ces bois, négligés par les Propriétaires, parce qu'ils étaient jadis de peu de valeur, ont

D 2 


\section{$(52)$}

été abandonnés aux Fermiers, qui pouvaient les couper au moins une fois dans le cours de leur bail. Ceux-ci en ont eu peu de foin; la réparation des clôtures eft coûteufe, ils s'en font difpenfé; \& loin de furveiller leur Pâtres, ils ont toléré qu'ils y conduifffent leur bétail; de forte que la plupart de ces boquetaux, anciennement de meilleure effence, ont infenfiblement dégénéré en aulnayes, dont le bétail rebute la feuille, \& qui ne dornent que des fagots peu eftimés.

D'autres Fermiers, pour avoir tout à la fois du fagotage \& un mauvais pâtis, ont converti leurs bois en tronchées, manière d'exploiter dont on ne faurait dire trop de mal. Enfin, lorfque la valeur de ces bois a été réduite prefque à rien, on s'eft déterminé à les défricher.

Le Cultivateur, en Breffe, fait beaucoup plus d'ufage de fagot que de gros brins. $I^{0}$.; pour le four : chaque domaine a le fien, \& les préparations du maïs confomment beaucoup de bois; $2^{\circ}$. pour la chambre chauffière, où toute la famille fe raffemble; la cheminée eft au milieu de cette chambre.Une large pyramide quadrangulaire, dont la pointe s'élève au-deffus du toit, reçoit la fumée; \& comme à la crémaillere pend une énorme chaudière où cuifent les raves, les courges, les farines pour l'engrais du bétail, \&x. on a befoin du feu vif \& clair que donne le fagot. 


\section{( 53 )}

C'eft ainfi que l'ufage de couper les bois tous les neuf ans s'eft établi parmi les Cultivateur's, \& voici comment il s'eft pareillement établi \& perpétué, même à l'égard des bois de réferve.

Indépendamment des Habitans, des Fermiers, \& des Grangers-Cultivateurs, les campagnes de Brefie font peuplées de Chambriers, c'eft-à-dire, de petits ménages ne poffédant rien, ou preique rien, tombés, ou prêts à tomber dans la mifère, lorfque les tems font malheureux \& que le travail leur manque. Ces Chambriers fe louent pendant la moiffon \& le tems du battage, \& travaillent à la journée en faveur de ceux qui veullent bien les employer pendant l'Hiver. Je conviendrai, même avec plaifir, que lorfqu'on les emploie. habituellement, on en trouve de très-fidèles. Cependant en général ils achètent le moins de bois qu'ils peuvent.

Lorfque le taillis eft très-jeune, le bétail y fais plus de mal que la maraude; lorfqu'il prend fix à fept ans, les maraudeurs y font plus de mal que le bétail: fi le taillis eft encore plus âgé, il donne lieu à une autre forte de délit qui n'eft pas toujours commis par les Chambriers. On a befoin d'un manche d'outil, de perches pour fufpendre le maïs, d'un effieu, d'un brancard, d'un étai : on va choifir, \& on abat le brin le plus droit $\&$. de la plus belle efpérance dans un taillis ancien,

$\mathrm{D}_{3}$ 


\section{(54)}

qui le trouve par-là d'autant plus promptement \& complettement dévafté, que les taillis anciens font plus rares. Voilà pourquoi les Propriétaires qui ont quelques taillis en réferve, pour peu que ces taillis foient éloignés de leur habitation, fe voient pour ainfi dire forcés des les abattre à neuf ans.

Si pour en tirer un meilleur parti, en les adminiftrant foi-même, fi pour les mieux clore, \& pour en fufpendre les coupes, on fe réfoud à retirer ces petits bois des domaines, comme le Fermier n'eft plus intéreffé à leur confervation, les délits s'y multiplient. Il faut donc, ou que les bois foient réunis en affez grandes maffes pour dédommager de la dépenfe d'un Garde, ou qu'ils foient affez près du Poffeffeur pour qu'on n'ofe y caufer du dégat fous fes yeux.

Ces divers obitacles rendent l'abus des anticipations des coupes d'une difficulté extrême a déraciner (I). Je n'ai trouvé qu'un moyen de

(1) On peut rigoureufement démontrer à quoi monte Ja perte caufée par cette anticipation. D'après ce que nous avons vu, (table première) la valeur d'un taillis coupé à 9 ans eft à la valeur du même taillis coupé à 18 , comme 3200 eft à 5184 . Mais attendu que la coupe fe ferait deux fois en 18 ans, la proportion fe trouve réduite au rapport de 2400 à $\xi 184$.

La dépouille d'un taillis paffablement confervé de l'âge 


\section{(55)}

diminuer en partie le mal dont elles font la fource, il m’a même affez bien réuffi : c'eft celui de mettre, dans le bail à ferme, une claufe expreffe par laquelle, en abandonnant la dépouille du taillis au Fermier, le Propriétaire fe réferve le droit de le faire couper lui même, \& d'y conferver telle quantité de brins qu'il jugera à propos. Cette coupe reffemble à une éclaircie faite un peu largement. Le facrifice de quelques journées de Manœuvres n'eft pas confidérable, la façon \& la fortie du bois abattu demeurant à la charge du Fermier. De cette forte il y apyrait affez de bois de petit fervice pour que chacun en trouvant chez foi, fût moins tenté de faire des incurfions chez autrui, \& que les taillis en réferve demeuraffent à peu-près intacts.

\section{Corollaire.}

Par-tout le Royaume peut-être, en Breffe très-certainement, \& fur-tout dans les environs

de. 9 ans fe vend à bon marché, dans la plus grande partie des cantons de la Breffe 12 liv. par coùpée, ou rog liv. par arpent, ou à raifon de $\mathrm{I}$ liv. 5 f. $6 \mathrm{~d}$ d. de reproduction annuelle par coupée. Or $2400: 5184:: 12$ :

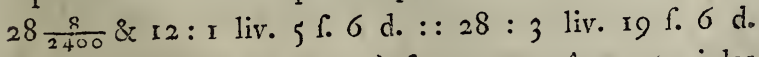
Il $\mathrm{y}$ a bien peu de terres à froment, même parmi les meilleures, qui puiffent s'affermer à ce prix.

$\mathrm{D}_{4}$ 
(56)

de Bourg, la confommation du bois excède évï demment la reproduction. Déja depuis longtems nous tirons la plus grande partie de nos bois de fervice, des montagnes de Bugey, les plus voifines, que nous dépeuplons de fapins; \& le prix auquel le bois de chauffage eft monté en peu d'années, comparé avec la hauffe proportionnellement plus faible qu'ont éprouvé les autres denrées, démontre, de la manière la plus allarmante la trifte vérité que j’annonce, non pour exciter des troubles, puifque nous tenons le remède en nos mains, mais pour inviter à l'ordre, \& provoquer la bien. faifance.

La cherté du bois, dira-t-on, depuis près de deux ans n'a pas fenfiblement augmenté. Je le veux : máis par quels moyens? Par un ravage affreux qui ruine l'efpérance future, par une dévaftation qui entraînera après elle de longs repentirs, par un pillage habituel, auquel le Gouvernement ne faurait apporter une attention trop prompte \& d'autant plus févère, que les mêmes plaintes, les mêmes réclamations s'élèvent dans tout le Royaume.

Tous les expédiens qui diminueront la confom. mation en la rendant plus économique, tous ceux qui favoriferont la reproduction par des femis, des plantations nouvelles, par la confervation \& la culture mieux combinée des anciennes planta* 


\section{( 57 )}

tions ne fauraient être négligés. Quelq̣ue faibles que l'on fuppofe, ou que foient fi l'on veut, chacun de ces:moyens en particulier, leur réunion leur imprimera de la force. J'en ai déja développé plufieurs dans un Mémoire fur les qualités individuelles des bois. Mais le moyen dont je n'avais point encore parlé, mais le plus puiffant de tous pour mettre la reproducion des bois en équilibre avec nos befoins, confifte dans la confervation rigide, \& le meilleur aménagement des forêts, devenues aujourd'hui à la difpofition nationale.

Les devoirs d'une grande adminiftration, les vues générales \& profondes qui déterminent fes décifions, ne font point circonfcrites dans les limites étroites où peut légitimement, où doit mêmefe renfermer un fimple Citoyen fage économe. Et telle économie qui, à l'égard d'un Père de famille, ferait digne de louange, pourrait devenir très-blamable dans un Adminiftrateur qui ne faurait pas la facrifier à un grand intérêt public, à un intérêt qui fe perpétuant d'âge en âge, préviendrait des malheurs de longue durée, où préparerait du moins une lente mais folide profpérité.

Relativement aux bois, relativement à cet objet de première importance, s'il n'eft pas de première néceffité, quel eft l'état ou nous fommes malheureufemeut tombés ? Et quel eft le point où il nous faut remonter? Je ne me lafferai 


\section{( 58 )}

point de le répéter, la confommation des bois excède la reproduction, \& il s'agit de les metre en équilibre; le peut-on? Oui, fans doute, fil l'on ne coupe point les bois nationaux avant qu'ils aient acquis le plus haut point d'accroiffement phyfique, le maximum fimple.

Pour le prouver, je ne reprendrai point les calculs que j’ai déja préfentés, qui annoncent que la quantité de bois que donne un taillis de trente ans, excède du double la quantité qu'eût donné ce même taillis à l'àge de 20; je ne propoferai point aux Economes que les Adminiftrateurs commettront à la régie des bois nationaux, je propoferai encore moins aux Adminiftrateurs eux - mêmes, d'aller le compas courbe à la main chercher la moyenne proportionnelle d'une portion de forêts, pour déterminer au jufte l'époque où il eft avantageux de la vendre. Non que cette opération foit ni longue ni pénible, ni de nature à laffer la patience françaife : mais c'eft une nouveauté, elle exige des foins, de l'exactitude, de l'attention, \& il peut être permis de fuppofer aux fousordres qui feraient néceffairement chargés de l'exécution, à-peu-près autant de préjugés que de lumières. Enfin l'expérience fût-elle mille fois plus facile encore, fi elle ne fe fait pas, ou fi elle fe fait mal, c'eft comme fi elle était impoffible. 


\section{(59)}

Mais en attendant que des Agronomes exercés. \& patiens, aient vérifiés, \& nous inftruifent fi la théorie que j’ai développée cadre avec leurs obfervations, je crois ne pouvoir mieux terminer cette première partie que par un apperçu rapide de la très-grande différence de L'INTÉRÊT DU Proprietétare qui coupe un bois pour le vendre, avec L'INTEREtT DU CONSOMMATEUR, c'eft-à-dire du Public.

Un Propriétaire poffède un taillis de 20 ans, \& de la valeur de 3000 livres; la production annuelle de ce bois doit donc être évaluée à I 501 .

Si le Propriétaire vend la dépouille de ce taillis à vingt ans, \& place à intérêts ces 3000 liv. ils augmenteront encore fon revenu de 150 liv.

S'il fufpend fa vente, \& que la valeur intrinfèque acquife par le taillis pendant la vingt-unième année, excède 300 livres, il a bien fait de fufpendre.

Si la valeur acquife pendant la vingt-unième année ne s'élève qu'à 250 livres, le Propriétaire eft en perte de 50 liv. mais la confommation a gagné 100 livres, c'eft-à-dire, le même terrain qui, fi le bois eût été coupé, n'eût reproduit qu'une valeur de I 50 livres, en a phyfiquement produit une de roo liv. de plus.

Dans ces circonftances peut-on forcer ce Propriétaire à fufpendre fa coupe ? Non, fans blef- 


\section{(60)}

fer SON INTÉRẼT, \& commettre une injuftice. Si un bois femblable était à la difpolition de la Nation, l'Adminiftrateur ferait-il bien d'en ordonner la coupe? Non, parce qu'il blefferait effentiellement L'INTÉRÊT DU CONSOMMATEUR, ou du Public, dont il eft le délégué \& le repréfentant.

Ainfi tout démontre, aux yeux de la raifon, l'extrême importance, ou plutôt l'abfolue néceffité, de porter la coupe des bois taillis nationaux à une époque beaucoup plus reculée que ne l'eft la coupe ordinaire des bois d'un particulier. Et après avoir combiné toutes les probabilités qu'on a pu raffembler jufqu'ici, j'eftime qu'on ne s'éloignera pas beaucoup du maximum qui a fait l'objet de nos recherches, en réglant à 30 ans la révolution de la coupe des bois nationaux, fitués fur un terrain profond \& fertile.

(r) Ce Mémoire a été lu par l'Auteur dans une Séance đu Confeil général du Département de i'Ain, \& l'Affemblée en rendant hommage aux talens \& au zèle de $M$. Varennede-Fénilie, a arrêté que copie de cet écrit ferait adreffée au Comité d'Agriculture \& de Commerce de l'Afremblée Nationale.

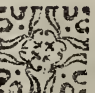




\section{(GI) \\ SECONDE PARTIE.}

\section{Bois de futaie.}

$1 \mathrm{E}$ в U T que fe propole un Agronome, en établiffant une futaie, eft d'obtenir la plus grande quantité de bois, dans le plus petit efpace, \& le moins de tems poffibles; ou, en d'autres termes, le meilleur aménagement des futaies eft en raifon compofée de la quantité, du tems \& de l'efpace.

D'où il fuit, $I^{\circ} \cdot Q u^{\prime} i l$ ne faut entreprendre d'établir des futaies, que fur un terrain profond \& fertile.

$2^{\circ}$. Qu'il eft à propos d'efpacer les arbres, de maniére que, fans qu'il y ait aucune place perdue, ils ne puiffent fe nuire réciproquement, ni ralentir mutuellement leur croiffance.

$3^{\circ}$. Qu'il ferait défavantageux, hormis le cas d'un fervice urgent \& néceffaire, de couper des arbres d'efpérance, fuffifamment efpacés, avant qu'ils aient acquis leur maximum individuel.

Ces propofitions exigent quelques dévelopemens.

On efpérerait en vain de voir de la futaie, fur un terrain qui n'auroit pas, au moins, deux pieds \& demi à trois pieds de profondeur de terre, propre à la végétation: les arbres y croîtraient avec trop de lenteur, \& feraient couronnés avant d'avoir acquis 


\section{(62)}

affez de groffeur pour fournir du bois de fervice. Un pareil fol n'eft bon que pour du taillis.

Cependant le mot fertile ne doit pas être entendu d'une manière trop générale; car, tel terrain, qui n'eft doué que d'une fertilité médiocre à l'égard des plantes céréales, produitfouvent de très-beaux bois; mais l'Agronome difcernera facilement cette fertilité relative, à l'effor qu'il verra prendre à fes taillis.

Les principes établis, quand nous avons traité de l'éclaircie des taillis, fur les diftances où doivent être les brins entre eux pour qu'ils puiffent profpérer, font également applicables aux futaies; car, ou provenues de femences, ou crûes fur fouche, elles ont néceffairement paffé par l'état de taillis avant d'être futaies.

Lorfqu'on coupe les taillis fréquemment, \& prefqu'entre deux terres, comme en Breffe, ils peuvent être aifément convertis en fuaties. Les fouches s'y renouvellent trop fréquemment, pour préfenter une large furface. Souvent, fur une portion de bois ancien, qui tient à une principale racine, éclattée \& défunie du tronc, il s'élance un jet vigoureux qui pouffe des racines au bourrelet, tandis que la vieille fouche, devenue ftérile, périt \& fournit de l'engrais.

Mais dans d'autres cantons du Royaume, \& affez fréquemment en Bourgogne, on voit des 


\section{$\left(6_{3}\right)$}

taillis dont les fouches trop efpacées forment audeffus du fol des brouffins, ou têtards, chargés de mouffe: le refte du terrain ne préfente ordinairement qu'une peloufe. Je crois cette manière d'exploiter les bois très-vicieufe. Elle eft, avec xaifon, profcrite par l'Ordonnance de 1769 , tit. $\mathrm{XV}$, art. 42. Des taillis auffi mal difpofés, fuffentils fur un terrain profond, donneront difficilement de la futaie; à moins qu'on ne fème des glands dans les intervalles, \& qu'on n'extirpe fucceffivement les fouches à mefure que les jeunes chênes auront crû fous leur abris.

J'eftime auff qu'il conviendrait d'ordonner à la prochaine coupe, l'abattis à fleur de terre de ces fouches proéminentes, \& d'en recouvrir les larges plaies avec du gazon; car on ne doit pas s'attentendre, quand même on fe bornerait à ne vouloir que du taillis, qu'il puiffe s'élever de beaux jets fur des brouffins à fibres dures \& contranchées; au lieu qu'il s'en dévelopera indubitablement fur les lèvres de ces mamelons recoupés \& couverts de terre, \& qu'il fe formera, comme on vient de le dire, des racines au bourrelet des nouvelles tiges.

On croit communément qưil y a perte réelle pour le Propriétaire qui veut élever une futaie fur taillis, \& que, s'il s'y détermine, c'eft un fa. crifice gratuit qu'il fait à fa pottérité. Il me pa- 


\section{(64)}

raît important de détruire ce préjugé: \& pou en démontrer la fauffeté.

Soit un arpent de taillis de l'àge de 20 ans en très-bon fond, portant, d'après les données de Duhamel à la diftance moyenne de 7 pieds 4 pouces, 900 brins de 20 pieds d'élévation, dont la croiffance ait été de 3 lignes de diamètre annuellement, \& dont la valeur foit égale à i 20 livres。 Par l'hypothèfe ces brins à 3 pieds de terre auront 5 pouces de diamètre.

Au lieu d'en faire la coupe blanche à la vingt. unième annẻe, il n'en fera fait l'éclaircie que d'environ moitié, dont la venté, ả la vérité, s'élévera au plus à 60 livres. C'eft le feul des facrifices à faire; mais le Propriétaire ne tardera pas à en être dédommagé, comme on le verra.

A la feconde révolution, c'eft-à-dire, à l'âge de quarante ans, cet arpent fera compofé de $45^{\circ}$ brins de 40 pieds de hauteur, portant Io pouces de diamètre à trois pieds de terre. On en coupera feulement 200 brins, dont la valeur fera de I92 livres. Car les cercles étant entre eux comme les carrés de leur diamètre, la fomme des carrés du diamètre de 900 brins de 5 pouces, eft à la fomme des carrés du diamètre de 200 brins de ro pouces, comme I 2500 à 20000 . Mais I 2500 font à I 20 livres, comme 20000 font à 192 livres; donc à la feconde révolution il y aura 72 livres 


\section{(65)}

de bénéfice fur le produit de la coupe ordinaire. Et l'on voudra bien obferver que nous n'avons pas ajouté à l'excédent de ce produit la hauteur acquife par les 200 brins, depuis l'âge de 20 ans jufqu’à 40.

A 60 ans, l'arpent contiendra 250 brins : il en fera coupé IIO. Ils porteront 15 pouces de diamètre à 3 pieds de terre, \& 9 à ro pouces de diamètre à la hauteur de 20 pieds. Ces brins feront en état, comme on le voit, de fournir de la petite charpente. Cependant nous n'y aurons aucun égard dans le calcul; nous continuerons à ne les eftimer que comme bois de chauffage, \& nous n'aurons également aucun égard à la hauteur acquife par de-là les vingt premiers pieds. On a cette proportion, I2500 : 120 livres : : 24750, fomme des carrés du diamètre des i io brins de I5 pouces : 237 liv. 12 fols. L'excédent fur le produit ordinaire fera de II 7 liv. 12 fols.

A 80 ans il fe trouvera 140 arbres : il en fera coupé moitié. Chacun de ces arbres ayant crû moyennement de 3 lignes de diamètre par an, fuivant l'hypothèfe, portera 20 pouces de diamètre à la hauteur de 3 pieds, \& pourra donner une pièce d'environ un pied d'équarriffage à fleur de terre, fur huit pouces d'équarriffage à la hauteur de 20 à 24 pieds; mais en continuant de n'évaluer la coupe que comme bois de chauffage, \& de 
(66)

n'eftimer, comme ci-devant; la hauteur de l'arbre' que de 20 pieds, nous avons cette proportion. I2500 : I 20 livres : : 28000 fomme des carrés du diamètre des 70 arbres de 20 pouces : 268 liv. I 6 fols. L'excédent fur le produit ordinaire eft de 148 livres 16 fols.

Sur quoi je prie d'obferver que les élémens de' mon calcul font dans tous les points beaucoup plus faibles que la nature ne les donne, puifque je n'ai porté le groffiffement moyen des brins qu'à 3 lignes de diamètre, ou à 9 lignes de tour annuellement, tandis que $M$. Tellès d'Acofta a obfervé, comme nous l'avons vu, qu'il étoit de I6 lignes de tour dans les très-bons terrains, que fuivant Buffon il eft de I2 à I 8 lignes fur un chêne vigoureux (I), \& que Duhamel \& moi (2)

(I) Hift. nat. fuppl. in $-4^{\circ}$. tom. 2 , page 113.

(2) J'ai fait éclaircir cet Hiver un taillis, eflence de chêne, de l'âge de $\mathrm{I} 3$ ans, fitué à trois lieues de la ville de Bourg, \& fur un bon fond. Il contient 180 coupées, ou environ 23 arpens. On a coupé à-peu-près moitié des brins. Cette éclaircie, faite avec foin fous la direction de mon Jardinier, m'a coûté un peu moins de 600 liv. de frais, \& m'a rendu un peu plus de 2400 liv. Le produit net de la feule éclaircie a donc été de 1800 liv. ou à raifon de $\sigma$ liv. de reproduction annuelle par arpent. Or la coupe entière de l'arpent de Duhamel n'eft évaluée que $\sigma$ liv. annuellement puifque $\frac{20}{120}=6$. J'ai laiffé fubfifter, comme on peut croire, les plus gros brins \& les 


\section{$(67)$}

2vons reconnu un groffiffement encore plus fort

plus élevés. Mais parmi ceux qui étaient abattus, j’en ai mefuré qui portaient 20 à 22 pieds d'élévation.

Le I Mai I79I, mon Jardinier a numéroté dans ce bois, \& mefuré avec un com as courbe à la hauteur de 3 pieds, feize chênes, cinq trembles, quatre aulnes, trois bouleaux, un orme \& un merifier. En tout trente brins.

Des feize chènes, fix ont été choifis parmi les plus gros, leur diamètre excédait 60 lignes : cinq parmi les noyens, leur diamètre était de 50 à 55 lignes ; \& cinq parmi les petits, dont le diamètre était de plus de 40 lignes. Les autres arbres ont été choifis parmi les moyens de leur erpèce.

Le groffffement moyen des feize chênes a été de $12 \frac{6}{15}$ lig. par an.

Celui des aulnes, d'environ ro lignes.

Les trembles avaient groffi annuellement de $12 \mathrm{lig}$.

Les bouleaux de $14 \frac{6}{\sqrt{3}}$ lignes.

L'orme de $10 \frac{2}{3}$ lignes

Et le merifier de $10 \frac{1}{\frac{1}{3}}$ lignes.

Il eft à remarquer quà chacune des éclaircies fuivantes, on laiffera fubfifter de préférence les plus gros brins; \& qu'ainfi, les arbres d'un tempérament faible, \& même les arbres moyens difparaiffant infenfiblement, le bois ne fera plus compofé que d'arbres vigoureux ' \& naturellement difpofés à une croiffance accéiérée. Je ne fais aucun doute que leur groffifement moyen n'augmente par la fuite, les éclaircies fucceffives laiffant à chacun d'eux plus de nourriture \& d'efpace. Je puis affurer que celle-ci produit déjà un effet agréable, \& je me propole de mettre mon fils en état de fuivre, après moi, cette expérience intéreffante. 
lorfque les arbres étaient efpacés largement ( 1 ):

A 100 ans on aura une futaie contenant 70 pieds d'arbres par arpent. Ils feront efpacés moyennement à la diftance de 26 pieds un quart, \& c'eft tout ce qu'un arpent peut en contenir, fil'on veut qu'ils acquièrent une belle groffeur. (Voyez la première partie de ce Mémoire, page 25 , note I. )

Conviendra-t-il à cet âge de couper cette futaie? Avant d'entamer l'examen de cette queftion, qu'il foit permis de faire une remarque.

Prefque tous les Officiers attachés aux Maìtrifes, qui ont écrit fur l'aménagement des forêts, ne ceffent de répéter que fans le balivage tout eft perdu, que la qualité du bois des baliveaux eft incomparablement fupérieure à celle du bois de futaie en maffif, qu'il eft plus dur, plus fort, \& particulièrement recherché par les Charpentiers de la Marine: Qu'au contraire le bois crû en maffif eft faible, mou, gras qu'il fe coupe comme de la rave; à peine, à les entendre, ferait-il bon pour les membrures d'une menuiferie. Ils donnent pour raifon de cette différence, qu'à chaque fois que le taillis qui environne les baliveaux a été abattu, le grand air \& les alternatives du froid

(I) Traitéde l'exploitation des bois, tom. I, page $4 \mathrm{I} 4 \mathrm{i}$ \& Traité des femis \& plantations, page 3 I 8. 


\section{(69)}

$\&$ du chaud, leur donne une force que n'acquiert jamais l'arbre qui a crû en maffif de futaie.

D'autre part, les Ecrivains les plus célèbres qui ont obfervé les bois en Phyficiens \& en Naturaliftes, qui ont à cet égard multiplié les ex. périences avec autant de foin 'que de fagacité, les Réaumur, les Duhamel, les Buffon, s'accordent à dire que les baliveaux font la perte des bois. Qu'on recoure fur-tout à l'ouvrage de Buffon, on y verra le détail des nombreufes expériences, $\&$ les raifons invincibles fur lefquelles ce célèbre Phificien avait fondé fon opinion ( $\mathrm{I}$ ).

Jai déjà remarqué comme unie contradiction bien étrange entre le fait \& les principes, l'aveu de $M$. Tellès d'Acofta, l'un des plus grands partifans des baliveaux, qui convient qu'en Champagne les Adjudicataires des coupes qui approvifionnent Paris, exploitent de préférence les baliveaux pour le chauffage, \& tirent peu à la charpente.

Veut-on encore un exemple plus fráppant des contradictions dont nos Ouvrages fur l'Agriculture fourmillent, lorfque leurs Auteurs fe jettent dans les généralités fans être prémunis d'un nombre fuffifant d'obfervations particulières ?

(x) Hiftoire Naturelle, fuppl. in $-4^{\circ}$. tom. 2 , pag. $11_{3}$ s 124, 128, 25 I, \&c. 


\section{( 70 )}

M. Plinguet, Ingénieur en chef de M. d'Orléanș, dans un Cuvrage imprimé en 1789 , fous le titre de Traité fur les réformations \& les aménagemens des forêts, où il eft principalement queftion de celles d'Orléans \& de Montargis, s'exprime dans les termes qui fuivent ( page 9).

“ Nous ne dirons rien fur la différence de bonté " qui fe trouve entre la charpente qui provient " des futaies, \& celle que produifent les baliveaux " fur taillis. D'une part, l'emploi des uns \& des " autres dans la conftruction ne nous démontre " que trop fouvent, par de mauváis effets, la dan: " gereufe qualité de ces derniers; \& d'autre part, " la théorie appuyée d'une fuite d'expériences, " faites avec infiniment de précautions par Buffon, " fur la force des bcis, ne laiffe aucun lieu " de douter de la mauvaife qualité de ceux-ci, " \& fera toujours préférer la charpente qui nous ". viendra des futaies".

Un Auteur prefque auffi moderne, M. Pannelier-d'Annel, dans une brochure intitulée : E/fai fur l'aménagement des forêts, imprimé en 1784 , nous dit; dans une note page 16 , ̀̀ l'occafion de la forêt de Compiègne; "Que les baliveaux fur " taillis ont des droits au moins fur un fens, que ". n'ont pas les arbres crûs en maffif de futaie, "qui, (page I4) ne viennent jamais droits". Propofition neuve, \& que $j$ 'ai lue plufieurs fois 


\section{( $\left.7^{1}\right)$}

pour vérifier fi je ne me trompais pas. " $\mathrm{Ce} q u i$ "fait, ajoute l'Auteur, que ces baliveaux donnent " des pièces dont la longueur répond à la hauteur " de l'arbre, des pièces de 30 à 60 pieds, \& " même quelquefois plus, \& comme arbresifolés, " continuellement frappés de l'air \& du foleil, ils " ont toute la qualité que comportent le climat " \& le terrain; en un mot ils ont ce qui manque " aux arbres venus en maffif de futaie, c'eft-à-

") dire tout ".

Le bois d'un baliveau, on en convient, eft plus dur \& plus denfe que celui d'un arbre fem. blable crû en maffif, dans le cas feulement où celui-ci aurait été gêné dans fa croiffance, \& qu'elle en eût été ralentie. Mais dans tous les caș il n'eft point vrai que le baliveau foit plus fort; puifquil eft très-chargé de nœuds, \& que les nouds affaibliffent le bois de plus d'un quart, comme le remarque Buffon (I). Nous croyons auffi que la plus grande denfité, ainfi que la plus grande dureté d'un arbre ifolé ne font point l'effet des caufes que rapportent les Sectateurs des baliveaux. Les coups de foleil fubits après une violente gelée, les alternatives de froid \& de

(i) Hiftoire Naturelle, fuppl. in-4 $4^{\circ}$. tom. 2 , pag. 128. Voyez auff Duhamel, Traité de l'exploitation des bois, tom. I, pag. 88.

E 4 


\section{(72)}

chaud, les violens orages, peuvent occafioner \& produifent en effet des gelivures, des chancres, des gouttière's, des roulures, des brifures, des accidens enfin qui détruifent la plus grande partie de nos baliveaux; mais nous ne voyons pas en quoi ils pourraient favorifer leur croiffance \& dunner du nerf à leur bois. Qui doute que l'air \& la lumière ne foient néceffaires à la végétation ? Mais ils ne fauraient avoir de l'influence fur la tige d'un arbre. Quel effet produiraient-ils au travers de l'épiderme épais \& fans vie, dont le corps de l'arbre eft environné ? C'eft fur les jeunes branches, les boutons \& les feuilles quils agiffent. Pour peu qu'on ait étudié la théorie de la végétation, on fait qu'il y a action \& réaction perpétuelles \& réciproques des feuilles fur les-racines, \& des racines fur les feuilles; \& qu'ainfi, pour qu'un arbre profpère, il lui faut un efpace fuffifant dans le fein de la terre pour y étendre fes racines, \& un efpace fuffifant dans le vague des airs pour y étendre fes rameaux. Toutes les fois que l'arbre eft gêné óu de l'une, ou de l'autre manière, fa croiffance en eft retardée, il s'affaiblit, il languit, \& quelquefois il en meurt. D'autre part, Buffon (I) \& Duhamel, nous ont appris que de deux arbres femblables; de

(1) Hift, Nat, fup. in-4ํ. t. 2, p. 124, \& Expl. des bois, p. 687. 


\section{(73)}

même âge \& crûs dans le même terrain, celui dont les couches annuelles font plus épaiffes \& le groffiffement plus accéléré eft incomparablement plus fort, plus nerveux \& fpécifiquement plus lourd que celui de l'arbre amaigri auquel on le compare. Ce n'eft donc point parce qu'un chêne aura crû en maffif de futaie qu'il eft faible, mais parce qu'il y a fouffert. Quoi qu'il en foit on pare à cet inconvénient par les éclaircies que nous propofons. Elles feront participer les arbres fucceffivement réfervés, tant aux bonnes qualités qu'on attribue aux baliveaux, qu'à celles des arbres crûs en maffif, \& les garantiront en même tems des défauts qu'on leur reproche à tous deux. Mais revenons à notre arpent de cent ans d'âge.

Les 70 arbres qui le compofent, dans l'hipothèfe, auront au moins 30 pieds de tige, portant I 5 pouces d'équarriffage au gros bout, \& 9 à ro pouces à l'autre extrémité, conformément à l'eftimation de Duhamel, qui a obfervé que les tiges diminuaient d'un pouce de gros par pied de hauteur. En cet état ils vaudraient au moins 8 liv. dans le Département que jhabite, \& par conféquent la valeur de l'arpent ferait de 1260 livres. Un coup d'œil jeté fur la table fuivante, prouvera l'avantage de l'aménagement que je propofe.

Si l'on eût continué de tenir cet arpent en taillis, il n'eût produit, dans le cours des cinq 


\section{( 74 )}

xévolutions, que 600 livres, mais au moyen des éclaircies, il a produit.

$\begin{array}{ccc}\text { A la première révolution. } 601 . " & \$ f . \\ \text { A la feconde il produira. } & 192 "\end{array}$

A la troifième..... 237 I2 7581.81 .

A la quatrième. .... 268 16

A la cinquième il vaudra. . . . . . I 260

Total en eftimant à bas prix. . . . $\overline{2018 \quad 8}$

On demande fi à cette époque il conviendra de l'abattre ? C'eft encore le calcul qui réfoudra cette queftion.

Nos fens, à moins d'ètre très-exercés, nous trompent, lorfque nous parcourons une futaie. Ce n'eft que par une approximation hafardée. que nous jugeons de la hauteur \& de la groffeur des tiges. Lorfqu'on paffe quelque tems fans vifiter un jeune taillis, on eft frappé en le revoyant du changement qu'il a éprouvé dans l'efpace de trois ou quatre années. Il n'en eft pas à beaucoup près de même d'une futaie; eût-on écé dix ans fans la parcourir, à peine s'appercevra-t-on que les tiges aient groffi. Plus les arbres étaient âgés à la précédente vifte, moins leur augmentation paraîtra fenfible; cependant elle eft réelle \& quelquefois très-forte. Mais ce n'eft qu'au, moyen d'un inftrument \& a l'aide du calcul, qu'on peut connaitre avec exactitude le volume \& la 


\section{( 75$)$}

valeur qu'ont acquis les tiges. (I) Car fuppofons que le diamètre d'un arbre, qui était de 25 pouces à l'àge de roo ans, ait augmenté de deux pouces $\&$ demi pendant les dix années fuivantes, à peine l'œil diftinguera-t-il cette augmentation. Cependant nous avons la proportion fuivante. 625 , carré $\mathrm{du}$ diamètre réduit en pouces carrés à IoO ans d'âge, font à $756 \frac{1}{4}$, carré du diamètre de I IOans, comme I 8 livres font à 2 livres is fols 10 deniers $\frac{2}{3}$. La différence eft de plus d'un cinquième en fus.

Le calcul démontre également, qu'à fuppofer que les arbres continuent de croitre uniformément \& qu'on en fufpende la coupe jufqu'à I 50 ans, la valeur augmente dans la proportion de I à $2 \frac{x}{4}$. Mais il eft à remarquer que lorfqu'un arbre excède les dimenfions communes, fon prix s'élève au-deffus des prix ordinaires. Par exemple : une pièce de bois de deux pieds d'équarriffage, fur 24 pieds de longueur, contient quatre fois autant de pieds cubes qu'en contient une pièce d'unè longeur femblable \& d'un pied d'équarriffage feulement; mais la rareté la fera certainement payer au de-là du quadruple.

Nous avons dit, page 29, que le maximum d'un

(I) A l'égard des futaies, je propoferais de fubftituer une chaîne platte \& bien graduée, au compas courbe, qui devient difficile à manier lorfqu'il eft d'un grand volume. 


\section{$(76)$}

arbre de futaie fe prolongeait jufqu'à l'inftant où cet arbre commence à s'altérer dans le cœur. Ce premier degré d'altération eft d'autant plus éloigné, que la croiffance de l'arbre a été plus accélérée. Ainfi, de deux chênes de même efpèce, fi l'un a acquis deux pieds de diamètre, je le fuppofe, pendant que l'autre n'aura pris que dix-huit pouces, le premier prolongera fa croiffance \& fa vie plus longtems que le fecond. C'eft le défaut de terrain \& d'efpace qui donne à celui-ci une vieilleffe \& un dépériffement anticipés.

Cependant tout a un terme dans la nature. L'arbre le plus vigoureux \& le plus avantageufement fitué, parvient enfin à la vieilleffe, \& de la vieilleffe il paffe à la décrépitude. C'eft dans le centre qu'il commence à s'altérer. Heureufement on reconnaît, à des fignes prefque certains, un commencement de dépériffement. Les branches de l'arbre meurent en partie à la cime, elle s'arrondit, fe couronne, le groffiffement de l'arbre diminue, fon fanage ceffe d'être auffr touffu, fes feuilles jauniffent plutôt; d'autres fignes annoncent également la vigueur d'un arbre, tels qu'une écorce unie, fine $\&$ d'une même couleur, des branches qui s'élèvent au haut de l'arbre, des feuilles étoffées à la cime, \& encore vertes fur la fin de l'Automne. Un grand ufage donne, à cet égard, aux Foreftiers un coup d'œil qui les trompe 


\section{( 77 )}

rarement. Quand la futaie commence à être fur le retour, on perdrait beaucoup à ne pas l'abattre. Il faut même fe hâter d'extirper les fouches \& de femer.

On doit donc établir, à l'égard du maximum des futaies, la même fous-divifion dont il a été fait mention à l'égard des taillis, favoir le maximum phyfique ou fimple, \& le maximum compofé, dans lequel il entre une donnée de plus, favoir lintérêt pécuniaire du prix de la vente.

Dans l'exemple que nous venons de rapporter, il. eft certainement de l'intérêt du confommateur que la coupe foit fufpendue jufqu'à l'époque de I 50 ans d'àge, à laquelle nous fixons, par fuppofition, le plus haut point de croiffance phyfique, ou le maximum fimple; puifque pendant les so dernières années, cet arpent a acquis une augmentation de valeur égale à I 575 livres, \& qu'il n'avait acquis qu'une valeur de I260 livres pendant les 100 années précédentes.

Mais ici l'intérêt du Propriétaire va encore fe trouver en oppofition avec celui du Confommateur, effet que nous avons déjà remarqué par rapport au maximum compofé du taillis : car il eft clair que fi le Propriétaire fe détermine à vendre fon arpent de l'âge de 100 ans 1260 livres, ce capital, joint aux intérêts pendant 50 ans, s'élèvera à 44 ro livres, tandis que fi la coupe du 


\section{( 78$)$}

même arpent eft différée pendant 50 ans de plus; elle n'équivaudra qu'à 2835 livres, perte à laquelle il faut encore ajouter celle de la croiffance pendant environ 50 ans du femis qui aurait été fait.

Mais les effets moraux compenferont en grande partie le réfultat du calcul arithmétiquement rigoureux que nous expofons. Une belle futaie eft le tableau le plus riche \& le plus impofant que puiffe offrir le règne végétal. Un Propriétaire ne fe prive jamais qu'à regret d'une auffi magnifique jouiffance, fur-tout fi fa futaie eft peu diftante de fon habitation. C'eft après avoir longtems réfíté que le befoin le détermine enfin d'y metre la coignée. On ne doit donc pas craindre qu'il calcule en Négociant la perte en arrérages d'intérêts qu'il éprouvera en fufpendant fa coupe: très-différent en cela de ces particuliers, qui fe réuniffant en compagnies pour une exploitation, dans laquelle ils ne confidèrent que le lucre, s'embarraffent affez peu des réclamations \& dư mécontentement qu'ils excitent autour d'eux.

Cependant, en fe bornant au calcul rigoureux; il eft démontré qu'en fuppofant que les corps ad: miniftratifs demeurent chargés de l'adminiftration des forêts nationales, il eft de l'intérêt de leurs commettans, c'eft-à-dire des Confommateurs \& du Public.

I. Que l'adminiftration tende à métamorpho. 


\section{( 79 )}

fer en futaies la plus grande quantité des taillis qui en feront fufceptibles.

$2^{\circ}$. Que l'abattage d'une forêt ne foit permis qu'après avoir reconnu que les arbres qui la compofent font parvenus à leur maximum individuel fimple.

Mais dans l'état préfent des chofes convient-il qu'un corps quelconque foit chargé de l'adminiftration économique des forêts nationales? C'eft la dernière \& la plus importante des queftions que nous avions à examiner.

Lorfque l'Affemblée Nationale décréta que les biens domaniaux \& eccléfiaftiques feraient aliénés, elle en excepta formellement les bois dont l'étendue excéderait cent arpens; avec la fage réferve de ne ftatuer fur les forêts d'une plus vafte étendue qu'après que du choc des opinions générales on aurait obtenu des réfultats qui la mettraient à portée de fixer fon opinion particulière $\&$ de prononcer.

La majorité a para d'abord incliner à ce que les grandes forêts fuffent gouvernées par des corps adminiftratifs. Mais la connaiffance des dévaftations qui fe font commifes dans ces derniers tems; a ramené beaucoup de perfonnes très-éclairées à l'opinion de la vente des forêts fans exception. Voici les motifs fur lefquels on fe fonde de part \& d'autre.

Les bois, difent les uns, font un genre de 


\section{$(80)$}

propriété qui, par fa nature, exige la réunion d'une vafte étendue : ce en quoi les bois diffèrent effentiellement des fonds cultivés, dont la divifion augmente affez fouvent le produit; au lieu que les frais pour la garde \& pour la clôture diminuent proportionnellement à mefure que la quantité des bois augmente. Sous ce point de vue il eft donc avantageux que la direçion en foit confiée à des corps adminiftratifs, puifquil en réfulte en général plus d'enfemble \& moins de frais.

D'autre part, comme le prix de la dépouille des bois excède fréquemment la valeur intrinféque du fol, qu'il peut même la décupler lorfqu'il eft queftion d'une futaie; fi l'on fe détermine à tout vendre, la Nation fera fans recours lorfque, les futaies fur-tout, dont la confervation eft l'objet de fes follicitudes, auront difparu. Le mal augmentera encore, \& fon effet fera plus prompt, fi des compagnies fe préfentent pour acquérir, \& fi elles font de leur acquifition un objet de fpéculation mercantile; or, comment l'empêcher? D'ailleurs l'acquéreur, quel quill foit, doit difpofer librement de fa propriété fuivant fon plus grand avantage, ou réel, ou imaginaire; on court donc, en vendant, le rifque d'être fous peu d'années dans une difette abfolue de bois pour la confruction civile \& pour la marine.

Ceux 


\section{(8r)}

Ceux de l'opinion contraire répondent qu'il eft impoffible qu'une inftitution humaine foit parfaite en tous fes points, \& que, puifqu'il n'exifte point de terme moyen entre le parti de vendre ou de ne vendre pas, il faut néceffairement pré. férer le parti qui préfente le moins d'inconvéniens.

Il s'eft fait, difent-ils, une horrible dévaftation depuis deux ans dans les forêts nationales; l'habitude du pillage s'eft contractée par une claffe d'hommes pour qui ce délit eft devenu l'unique occupation. Ce gain illicite, qu'ils préfèrent aujourd'hui, comme plus lucratif au falaire qui leur eft offert dans les atteliers ordinaires, les portera toujours à confidérer comme un bien propre ces bois, tant qu'ils continueront d'être réputés nationaux. Le défordre non réprimé augmente la licence; plufieurs malfaiteurs vont aujourd'hui avec des voitures \& à main armée, qui précédemment fe contentaient de porter une charge fous laquelle on les voyait quelquefois fuccomber. Les Directoires ont fait jufqu'ici, \& vraifemblablement feront par la fuite, d'inutiles efforts pour arrêter ce brigandage. Le paffé eft malheureufement à cet égard le garant de l'avenir.

On ne peut guère reprocher, ajoutent-ils, à l'Ouvrage du grand Colbert, à la fameufe Ordonnance des Eaux \& Forêts, que quelques erreurs

$$
\text { F }
$$


(82)

en phyfique, mais c'était la phylique du tems, fans toucher aux parties effentielles d'une Ordon. nance qui, malgré fes défauts, n'en eft pas moins un chef-d'cuvre de précaution \& de rédaction. Cependant que d'abus fe font introduits fous fon ombre! Si la vigilance des Officiers des Maîtrifes, fi l'intérêt que chacun d'eux en particulier, depuis le grand Maitre jufqu'aux Gardes, avait à la rigoureufe exécution de l'Ordonnance de 1669 , fi l'hiérarchie de leur mutuelle furveillance n'ont pû empêcher que lès bois du domaine du Roi, \& fur-tout des communautés villageoifes, ne fuffent les plus mal adminiftés du Royaume, comment fe flatter que des corps adminiftratifs quelconques puiffent, avec encore moins d'autorité $\&$ d'expérience, furveiller l'exécution de la loi nouvelle qui interviendra ? On la rectifiera fur l'ancienne, on la fimplifiera fans doute; mais elle n'en exigera pas moins des connaiffances foreftières de la part du furveillant choifi pour la faire exécuter. A qui les Diftrifts, les Départemens, ou le Gouvernement accorderont-ils cette furveillancefur une maffe de propriétés augmentée par la maffe encore plus forte des forêts qui appartenaient ci-devant aux communautés religieufes \& aux chefs de l'églife ? Que de connaiffances à acquérir par le Commiffaite qui en fera chargé ! Et quand il les aura acquifes on 


\section{( 83$)$}

lui donnera un fucceffeur. $\mathrm{Si}$ on ne lui en. donne point, l'abus ne tardera pas à renaître de fa cendre.

Ce qui eft poffédé en commun, difent-ils encore, n'eft véritablement poffédé par perfonne. L'intérêt eft trop divifé parmi un grand nombre de co-partageans pour que la confervation foit par eux efficacement furveillée, \& il n'eft point affez actif de la part d'un fimple prépofé, quelque zèle \& quelques lumières qu'on lui fuppofe, pour équivaloir à l'intérêt perfonnel d'un Propriétaire. L'état déplorable enfin où font tombés les bois communaux, font un pronoftic fâcheux fur l'état futur des bois nationaux, s'ils ne font pas aliénés.

Telle eft la réplique de ceux qui votent pour la vente, à l'argument des motionaires qui s'y oppofent. Mais n'eft-il pas de préfervatifs contre les abus que ceux-ci redoutent? Or sil en exifte la queftion eft décidée. C'eft de la recherche de ces moyens que s'eft principalement occupée la Société Royale d'Agriculture, dont les travaux ne doivent tendre qu’à éclairer fur les progrès d'un Art devenu aujourd'hui notre première reffource.

Pour trouver le correctif que l'on cherche, il eft néceffaire de remonter aux principes qui différencient effentiellement l'économie foreftière $\mathrm{F} 2$ 


\section{( 84$)$}

de l'économie des champs cultivés, principes auxquels il nous femble qu'on n'a pas fait encore fuffifament d'attention, \& dont les effets font it diffemblables qu'ils peuvent à peine fe comparer.

Tout eft avances, art \& travail perpétuels fur les fonds en culture. L'intérêt de ces avances, joint au prix du travail, monte le plus fouvent aux quatre cinquièmes de la valeur reproduite, $\&$ fe renouvelle indifpenfablemènt après chaque récolte.

Les bois au contraire exigent peu de frais; on les y renouvelle du moins fi rarement, qu’à dire vrai c'eft la Naturé qui fait tout : à peine l'Art lui prête-t-il quelques fecours; encore eít-ce principalement à ne pas gêner la Nature que cet Art confifte; première différence.

Le moment de la récolte des plantes céreales eft fixe, \& déterminé par celui de la maturité. auffi font-elles rarement dévaftées par les hommes, parce que pendant les moiffons tous font occupés. Il n'en eft pas tout à fait de même, à la vérité, de certains fruits de la terre qui n'exigent pas une parfaite maturité pour être déjà commeftible, tels que le raifin, le maïs, les raves, les pommes-de - terre, \&c. Cependant s'il s'en dérobe une partie, la perte n'eft qu'accidenrelle \& ne porte que fur le produit d'une feule année. 


\section{$(85)$}

Mais les bois nont pas de maturité proprement dite, à tout âge leur dépouille a une valeur quel. conque. Ainfi, \& indépendamment du mal incalculable qu'y caufe lá vaine pâture, l'Eté comme lHiver, la nuit comme le jour, tous les tems font boris pour les maraudeurs; \& la perte quils caufent - eft d'autant moins réparable, qu'elle enlève le produit d'une plus longue attente; deuxième différence.

Comme la, valeur intrinfèque du fond cultivable aliéné demeure à-peu-près la même, le Fermier ou le Métayer euffent-ils été négligens, le Vendeur court peu de rifques à laiffer à fon Acquéreur plus ou moins de délai pour s'acquitter; puifque, fi les conditions de l'áliénation font mal exécutées, il lui refte la faculté de rentrer dans fon ancienne propriété, qui n'a pas fenfiblement perdu de fa valeur première.

$\mathrm{Au}$ lieu que la valeur d'une futaie fur-tout, étant quelquefois décuple de la valeur intrinfèque du terrain qui l'a produite, il eft jufte \& naturel qu'en l'aliénant, le Vendeur exige le prix en entier de fa vente, ou du moins un cautionnement équivalent : fans quoi, \& fi la futaie eft abattue, il ne refte plus de recours contre un Acquéreur de mauvaife foi, ou diffipateur, ou feulement mauvais économe; troifième différence.

Nous en àvons encore une effentielle à remar- 


\section{( 86 )}

quer entre la vente faite par un particulier \& la vente faite par la Nation.

Un particulier qui a reçu le prix de fa forêt vendue, eft auffi indifférent fur l'úfage que l'Acquéreur fera de fa propriété que fi elle ne lui eût jamais appartenue; mais fi c'eft la Nation qui vend on ne faurait dire qu'elle demeure fans intérêt, même en fuppofant le prix de la vente totalement acquitté; parce que le meilleur aménagement poffible de la chofe aliénée importe au Public confommateur. Il eft donc jufte d'impofer à l'Acquéreur des conditions confervatrices, qui le forcent, en quelque forte, d'adminiftrer le bien qui lui eft tranfmis d'une manière qui lui foit profitable \& tout à la fois avantageufe à la Nation. L'on ferait mal fordé à foutenir qu'un pareil acte gêne la liberté, puifque l'Acquéreur a été parfaitement libre d'en accepter ou d'en refufer les conditions lorfqu'il a tranfigé; \& que d'ailleurs une loi n'eft point dure lorfqu'elle n'enlève à celui qui s'y foumet que la faculté d'abufer.

D'après ces principes, auxquels les bornes d'un Mémoire ne permettent pas de donner plus d'étendue, nous eftimons que l'aliénation fans réferve \& à perpétuité des forêts nationales, \& fans diftinction de taillis ou de futaie, faite, fur-tout, à deniers comptans, fera avantageufe à la Nation, \& qu'elle 


\section{( 87 )}

éteindra un immenfe capital dont les intérêts fons hors de toute proportion avec le revenu qu'elle retirerait en adminiftrant elle-même fès forêts.

C'eft d'après ce Mémoire, dont nous adoptons les principes \& les conféquences, que nous penfons qu'il ferait utile :

Que l'aliénation des forêts \& des bois nationaux fùt faite fans diftinction de taillis \& de futaies:

Que l'Acquéreur d'un taillis ne pût couper les baliveaux, qu'en laiffant en réferve dans le même taillis, ou dans tout autre dont il ferait propriétaire, un arpent croître en futaie, par fix arpens dont les baliveaux auraient été abattus:

Qu'aucun défrichement, dans les bois nationaux acquis, ne pût être fait qu'en affectant en remplacement un bois nouvellement femé ou planté, équivalant à la partie défrichée :

Que tout Acquéreur de taillis \& de futaies, ne pût les aliéner en tout ou en partie, qu'en ftipulant que fon Acquéreur immédiat fera tenu de pofféder \& de jouir aux conditions portées par l'adjudication defdits bois \& futaies, \& de no. tifier l'acte de vente au Direqoire du Dittria \& du Département.

Signé, ABEILLE, l'AbbéLEFEBVRE, THOUIN。 


\section{(88)}

EXtRaIt des Regiftres de la Société Royale d'A. - griculture, du 22 Mai I79I.

La Société Royale d"Ag'ticulture convaincue que le dépériffement des forêts du Royaume - devient de plus en plus allarmant, que la confommation des bois deftinés au chauffage, à la charpente \& à la marine, excède la reproduction, que la néceffité de veiller à leur confervation, à leur accroiffement \& à un meilleur aménagement fe fait fentir plus que jamais, \& particulièrement pour ce qui concerne les forêts Nationales, perfuadée qu'il eft de fon devoir \& conforme à lefprit de fon inftitution de donner fon opinion fur un objet d'un intérêt auffi général, \& d'en développer les motifs, avait chargé MM. Abeille, Lefebvre \& Thouin, de lui préfenter un travail particulier fur cet objet. La Compagnie après avoir entendu le rapport de fes Commiffaires \& l'avoir difcuté dans plufieurs de fes Séancëss, en a adopté le contenu, \& $a^{\prime}$ décidé qu'une copie en ferait préfentée à l'Affemblée Nationale, \& que cet écrit ferait imprimé à fes propres frais, pour être diftribué.

\section{Certifiéconforme à l'original,}

A. BROUSSONET, Secrétaire perpétuel. 


\section{$E R R A T A$.}

$\mathrm{P}_{\mathrm{AGE}} \mathrm{I5}_{5}$, ligne 9 de la note, l'autre de $\mathbf{3}$, lifez, l'autre de 16.

Page 20, lig. 12 , tous, lif. tout.

Page 25, lig. 14 \& 17 de la note, valon, lif. vallon.

Page 27, ligne dernière de la note, afféné, de bas en haut; fupprimez la yirgule qui fait contrefens.

Page 28, première ligne de la note, fus un brin, lif. fur un brin.

Page 28 , lig. 1o, chaque bois, lif. chaque brin.

$P_{0}$ ge 29, ligne pénultiène, dans ce Mémoire, lif. dansla feconde partie de ce Mémoire.

Page 32 , lig. I \& 2, 9 la vingt-troifième, lif. 9 lignes à la vingt-troifième.

Page 32 , lig. 6, donc, lif. dont.

Page 33 , lig. 14 , donc, lif. dont,

Page 34, lig. 8, formule du cacul, lif: formule du calcul. Page 39 , lig. 4, cacul, lif. calcul.

Page 39, lig. 23, cacul, lif. calcul.

Page 52 , lig. 2I, chambre chauffière, lif. chambre chauffure: Page 62, lig. I9, fuaties, lif. futaies.

Page 63, lig. 6, 1769, lif. 1669.

Page 82, lig. première, après les mots phyfique du tems, ajoute,$\&$ ces erreurs peut-être étaient faciles à rectifier: 


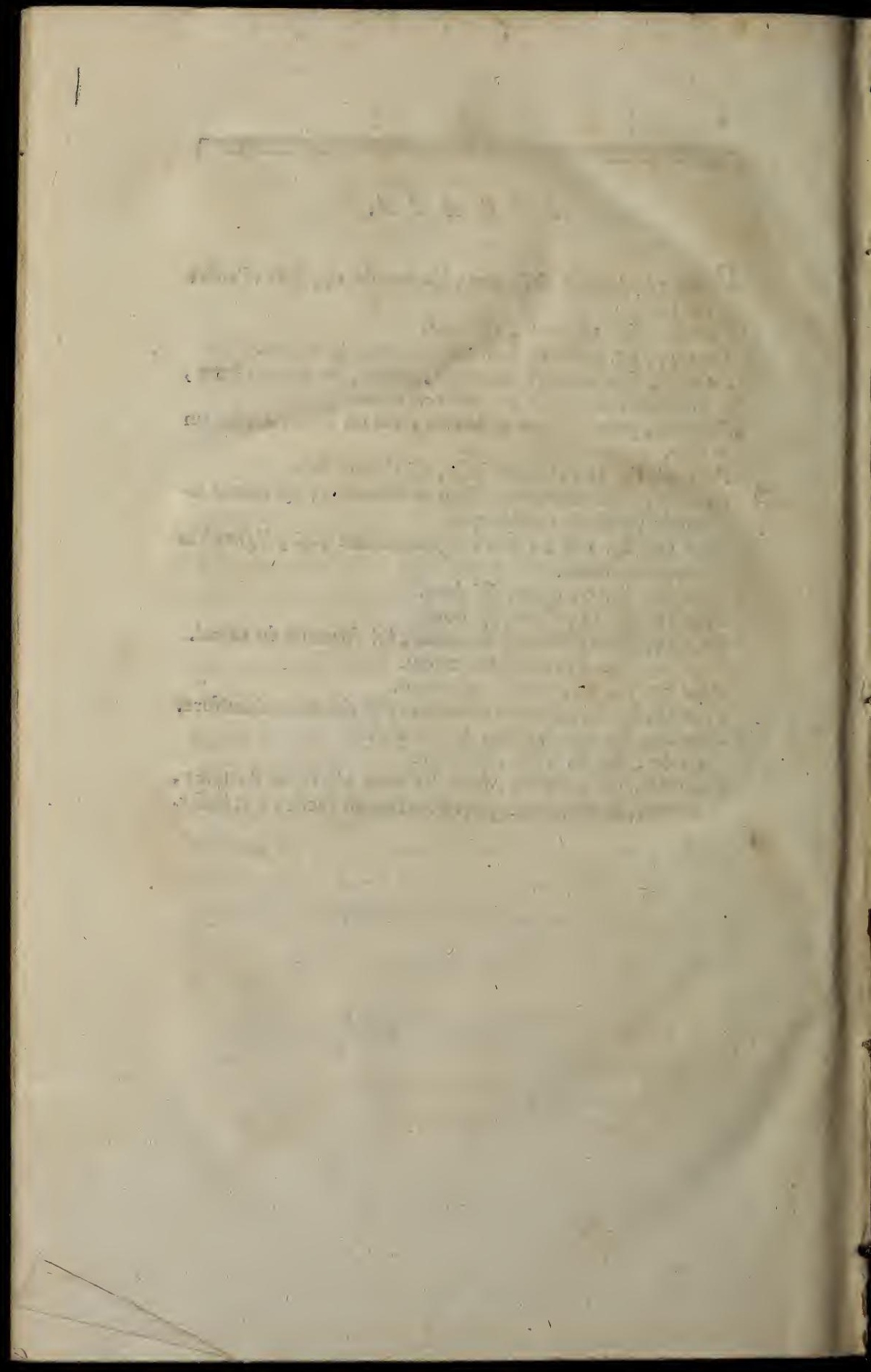

